Pacific Journal of Mathematics

ON COMPACTIFICATIONS OF METRIC SPACES WITH 


\section{ON COMPACTIFICATIONS OF METRIC SPACES WITH TRANSFINITE DIMENSIONS}

\section{LEONID LUXEMBURG}

In this paper we prove that every separable metric space $X$ with transfinite dimension Ind $X$ has metric compactification $c X$ such that

$$
\text { Ind } c X=\operatorname{Ind} X \text {, ind } c X=\text { ind } X, D(c X)=D(X) \text {, }
$$

where ind $X$ (Ind $X$ ) denotes small (large) inductive transfinite dimension, and $D(X)$ denotes the transfinite $D$-dimension. More generally, let $T$ be a set of invariants (ind, Ind, $D$ ). We consider the following problem:

Let $R \cong T$ and $X$ be a metric space. Does there exist a bicompactum (complete space) $c X \supset X$ such that

$$
\mu(X)=\mu(c X) \text { for } \mu \in R \text {. }
$$

When it is not so, we give counterexamples. We give also necessary and sufficient conditions of the existence of transfinite dimensions of separable metric space in terms of compactifications.

o. Introduction. In this paper we consider three transfinite invariants: ind $X$, Ind $X, D(X)$ where ind $X$ (respectively, Ind $X$ ) is small (respectively large) transfinite inductive dimension and $D(X)$ is $D$-dimension, see [3], Henderson.

Definition 0.1. (a) ind $X=-1 \Leftrightarrow X=\varnothing$.

(b) We assume that for every ordinal number $\alpha<\beta$ the class of spaces $X$ with ind $X \leqq \alpha$ is defined. Then ind $X \leqq \beta$ if for every point $x \in X$ and a closed subset $F, x \notin F \subset X$, there exists a neighborhood $O_{x}$ of $x$ such that

$$
\begin{aligned}
& O_{x} \subset X \backslash F \\
& \text { ind } F r O_{x} \leqq \alpha<\beta_{\mathfrak{I}} .
\end{aligned}
$$

We put ind $X=\min \{\beta$ : ind $X \leqq \beta\}$.

(c) The dimension $\operatorname{ind}_{x} X$ of a space $X$ in a point $x \in X \leqq \beta$ if there exists a base $\left\{O_{\lambda}, \lambda\right\}$ in this point, such that

$$
\text { ind } \mathrm{FrO}_{\lambda}<\beta \text {. }
$$

We put $\operatorname{ind}_{x} X=\min \left\{\beta: \operatorname{ind}_{x} X \leqq \beta\right\}$.

${ }^{1} \operatorname{Fr} A$ denotes the boundary of $A$. 
Definition 0.2. (a) Ind $X=-1 \Leftrightarrow X=\varnothing$.

(b) Let for every ordinal number $\alpha<\beta$ the class of spaces $X$ with Ind $X \leqq \alpha$ is defined. Then, Ind $X \leqq \beta$ if for every pair of disjoint closed subsets $F$ and $G$ there exists a partition ${ }^{2} C$ between $F$ and $G$ such that

$$
\text { Ind } C \leqq \alpha<\beta
$$

We put

$$
\text { Ind } X=\min \{\beta: \text { Ind } X \leqq \beta\} \text {. }
$$

We note that the dimension ind we can also introduce using partitions, because if $x \in \bar{V} \subset X \backslash F$ and $V$ is open, then $F r V$ is a partition between $x$ and $F$.

Let us introduce some notations. For every ordinal number $\beta$ the equality $\beta=\alpha+n$ holds, where $\alpha$ is a limit number or 0 , and $n=0,1,2, \cdots$. Then we set

$$
K(\beta)=n, \quad J(\beta)=\alpha .
$$

Definition 0.3. See [3], Henderson. We put $D(\phi)=-1$. If $X \neq \varnothing$ then $D(X)$ is the smallest ordinal number $\beta$ such that there exists a collection of sets

$$
\left\{A_{\xi}: 0 \leqq \xi \leqq \gamma\right\}
$$

satisfying the following conditions:

(a) $X=\cup\left\{A_{\xi}: 0 \leqq \xi \leqq \gamma\right\}$.

(b) Every set $A_{\xi}$ is closed and finite dimensional.

(c) For any $\delta \leqq \gamma$ the set

$$
\cup\left\{A_{\alpha}: \delta \leqq \alpha \leqq \gamma\right\} \text { is closed in } X \text {. }
$$

(d) $J(\beta)=\gamma$, Ind $A_{r} \leqq K(\beta)$.

(e) For any point $x \in X$ there exists the greatest number $\delta \leqq \gamma$ such that $x \in A_{\dot{\delta}}$.

If there is no such number $\beta$ we put $D(X)=\Delta$, where $\Delta$ is an abstract symbol such that $\Delta>\beta$ for any ordinal number $\beta$ and $\Delta+$ $\beta=\beta+\Delta=\beta \times \Delta=\Delta \times \beta=\Delta$.

If conditions (a)-(e) hold then equality (a) is called a $\beta-D$ representation of a space $X$.

Hence, for any space $X$ the dimension $D(X)$ is either an ordinal number or the symbol $\Delta$.

For any compact metric space $X$ having dimension Ind $X$

${ }^{2}$ A partition $C$ in $X$ between sets $A$ and $B$ is a closed set in $X$ such that $X \backslash C=$ $U \cup V, U \cap V=\varnothing, A \subset U, B \subset V$, for some open in $X$ sets $U$ and $V$. 


$$
\text { Ind } X \leqq D(X)
$$

(see [3], Henderson). For further results concerning $D$-dimension see [11], Luxemburg. Inequalities

$$
\text { ind } X \leqq \operatorname{Ind} X
$$

and

$$
D(X) \leqq D(Y) \text {, ind } X \leqq \operatorname{ind} Y \text { for } X \subset Y
$$

are evident for all topological spaces. However, the dimensions ind $X$ and Ind $X$ are not defined for every space. For example Hilbert cube $I^{\omega}$ has no inductive transfinite dimensions [5], p. 51 (Hurewicz and Wallman). Let

$$
Z=\bigcup_{n=1}^{\infty} I^{n}
$$

be the discrete union of Euclidean cubes $I^{n}$. Then obviously, ind $Z=$ $\omega_{0}$. However the dimension Ind $X$ does not exist. But obviously

(2) if for a space $X$ the dimension Ind $X$ exists, then the dimension ind $X$ also exists ${ }^{3}$.

There are compact metric spaces $X$ such that ind $X<$ Ind $X<D(X)$, see [12], [13], Luxemburg.

In what follows all spaces are assumed to be metric and all mappings to be continuous if otherwise is not stated.

A space $X$ we call finite dimensional if $\operatorname{dim} X \leqq n$ for some $n=$ $-1,0,1,2, \cdots$, where $\operatorname{dim} X$ is the covering dimension 4 . For finite dimensional metric space $X$

$$
D(X)=\operatorname{Ind} X=\operatorname{dim} X
$$

and

$$
D(X)=\operatorname{Ind} X=\text { ind } X=\operatorname{dim} X
$$

if $X$ is separable.

Definition 0.4. For any space $Z$ we denote by $P(Z)$ a closed subset such that $Z \backslash P(Z)$ is the union of all finite dimensional sets, open in $X$. For spaces $X$ and $Y$ with $\operatorname{diam} Y<\infty$ we denote by

${ }^{3}$ For compact metric space $X$ the dimension ind $X$ exists if and only if the dimension Ind $X$ exists (see [22], Smirnov).

${ }^{4} \operatorname{dim} X \leqq n$ if for any open covering $U$ of a space $X$ there exists an open refinement $V$ of $U$ having the order $\leqq n+1$, i.e., the intersection of any $(n+2)$ elements of $V$ is empty. 
$C(X, Y)$ the space of all continuous mappings $f: X \rightarrow Y$ with the metric $d\left(f_{1}, f_{2}\right)=\sup \left\{\delta\left(f_{1}(x), f_{2}(x)\right): x \in X\right\}$, where $\delta$ is the metric on $Y$. We note that the space $C(X, Y)$ is complete if $Y$ is complete. We consider a Hilbert cube $I^{\omega}$ to be the set of all sequences $\left\{x_{i}\right\}, 0 \leqq$ $x_{i} \leqq 1$, with the metric, defined by the equality

$$
d\left(\left\{x_{i}\right\},\left\{y_{i}\right\}\right)=\sum_{i=1}^{\infty}\left|x_{i}-y_{i}\right| \cdot 2^{-i}, \quad i=1,2, \cdots .
$$

The number $x_{i}$ is called the $i$-coordinate of a point $x=\left\{x_{i}\right\} \in I^{\omega}$.

1. On homeomorph mappings to the Hilbert cube.

THEOREM 1.1. (The Compactification Theorem). Let $X$ be a separable space and a fixed countable system of closed sets $L_{i}$, $(i=$ $1,2, \cdots)$ such that dimensions Ind $L_{i}$ exist.

Then the set $\Psi$ of all homeomorphisms $f: X \rightarrow I^{\omega}$ of the space $X$ to the Hilbert cube $I^{\omega}$ such that for each $i$ the equalities

(a) Ind $L_{i}=$ Ind $\overline{f\left(L_{i}\right)}$

(b) ind $L_{i}=$ ind $\overline{f\left(L_{i}\right)}$

(c) $D\left(L_{i}\right)=D\left(\overline{f\left(L_{i}\right)}\right)$

(d) $f(P(X))=\overline{P f(X)}$

are satisfied contains an everywhere dense set of type $G_{\delta}$ in the space $C\left(X, I^{\omega}\right)$.

In the case when the dimensions Ind $C_{i}$ are finite, this theorem has been proved in [8], Kuratowski. See also [14], Luxemburg for infinite dimensional case.

CoROLLARY 1.1. For any separable space $X$ having dimension Ind $X$ there exists a separable compactification $X$ such that

Ind $c X=$ Ind $X$, ind $c X=$ ind $X, D(c X)=D(X), P(c X)=P(X)$.

Proof. It is sufficient to put in Theorem 1.1

$$
L_{i}=X, \quad c X=\overline{f\left(L_{i}\right)} .
$$

For the proof of this theorem we need some preliminary lemmas.

Lemma 1.1. Let $A$ and $B$ be two fixed closed sets in the space $X$ and put

$$
C=A \cap B
$$

Then for any $n=1,2, \cdots$ the set $\phi_{n}$ of mappings $f$ from the space 
$X$ to a compact space $Z$ such that

$$
\overline{f(A)} \cap \overline{f(B)} \backslash O_{1 / n} \overline{f(C)}=\varnothing^{5}
$$

is open in $C(X, Z)$.

Proof. We suppose that the set $\phi_{n}$ is not open in $C(X, Z)$. Then for some $f \in \phi_{n}$ there exists a sequence of mappings $g_{k} \in C(X, Z) \backslash \phi_{n}$, $k=1,2, \cdots$, such that

$$
\lim _{k \rightarrow \infty} g_{k}=f
$$

and

$$
\overline{g_{k}(A)} \cap \overline{g_{k}(B)} \backslash O_{1 / n} \overline{g_{k}(C)} \neq \varnothing .
$$

Therefore for any $k$ there eixsts a pair of points $a_{k} \in A, b_{k} \in B$, such that

$$
\begin{aligned}
& \delta\left(g_{k}\left(a_{k}\right), g_{k}\left(b_{k}\right)\right)<\frac{1}{k}, \delta\left(g_{k}\left(a_{k}\right), \overline{g_{k}(C)}\right) \geqq \frac{1}{n}-\frac{1}{k} \\
& \delta\left(g_{k}\left(b_{k}\right), \overline{g_{k}(C)} \geqq \frac{1}{n}-\frac{1}{k}\right.
\end{aligned}
$$

where $\delta$ denotes a metric on $Z$. Since $Z$ is a compactum there exists a point $p \in Z$ such that

$$
p=\lim _{k_{i} \rightarrow \infty} g_{k_{i}}\left(a_{k_{i}}\right)=\lim _{k_{i} \rightarrow \infty} g_{k_{i}}\left(b_{k i}\right)
$$

for some subsequence of integers $k_{i}$. Since $\lim _{k_{i} \rightarrow \infty} g_{k i}=f$ we have

$$
p=\lim _{k_{i} \rightarrow \infty} f\left(a_{k_{i}}\right)=\lim _{k_{1} \rightarrow \infty} f\left(b_{k_{i}}\right) .
$$

Consequently

$$
p \in \overline{f(A)} \cap \overline{f(B)} .
$$

Let us show that

$$
\delta(p, \overline{f(C)}) \geqq \frac{1}{n}
$$

where $\delta(p, \overline{f(C)}=\inf \{\delta(p, x): x \in \overline{f(C)}\}$.

We suppose on the contrary. Then there eixsts a point $q \in C$, satisfying the condition:

$$
\delta(f(q), p)<\frac{1}{n}-\varepsilon
$$

${ }^{5} O_{\varepsilon} \mathscr{M}$ denotes $\varepsilon$-neighborhood of the set $\mathscr{M}$. 
for some $\varepsilon>0$. By virtue of (2), (5) we can find an integer $k_{i}$ such that

$$
d\left(g_{k i}, f\right)<\frac{\varepsilon}{4}, \delta\left(p, g_{k_{i}}\left(a_{k_{i}}\right)\right)<\frac{\varepsilon}{4}, \frac{1}{k_{i}}<\frac{\varepsilon}{2} .
$$

From (3), (8), (9) it follows that

$$
\begin{aligned}
\frac{1}{n}-\frac{1}{k_{i}} & \leqq \delta\left(g_{k_{i}}\left(a_{k_{i}}\right), g_{k_{i}}(q)\right) \\
& \leqq \delta\left(g_{k_{i}}\left(a_{k_{i}}\right), p\right)+\delta(p, f(q))+\delta\left(f(q), g_{k_{i}}(q)\right) \\
& <\frac{\varepsilon}{4}+\frac{1}{n}-\varepsilon+\frac{\varepsilon}{4} \\
& =\frac{1}{n}-\frac{\varepsilon}{2} .
\end{aligned}
$$

Consequently $1 / k_{i}>\varepsilon / 2$, which contradicts the condition (9). Thus, condition (7) holds. Consequently from conditions (6), (7) it follows that

$$
p \in \overline{f(A)} \cap \overline{f(B)} \backslash O_{1 / n}(\overline{f(C))},
$$

which contradicts condition (1). Hence the set $\phi_{n}$ is open in $C(X, Z)$. Since obviously

$$
\phi=\bigcap_{n=1}^{\infty} \phi_{n}
$$

we obtain that $\phi$ has type $G_{\delta}$ in the space $C(X, Z)$.

LEMMA 1.2. If in Lemma 1.1 we take $Z=I^{\omega}$, then the set $\phi$ of all mappings $f: X \rightarrow I^{\omega}$ such that

$$
\overline{f(A)} \cap \overline{f(B)}=\overline{f(C)}
$$

is everywhere dense $G_{\delta}$-set in $C\left(X, I^{\omega}\right)$.

Proof. First we show that the set $\phi_{n}$ is everywhere dense in $C\left(X, I^{\omega}\right)$. Let $f: X \rightarrow I^{\omega}$ be an arbitrary mapping and $\varepsilon>0$. We can find an integer $k$ such that

$$
2^{-k}<\frac{1}{4 n}, 2^{-k}<\varepsilon .
$$

Let us define a mapping $g: X \rightarrow I^{\omega}$, such that

$$
\delta(f, g)<\min \left[\frac{1}{4 n}, \varepsilon\right] \text {. }
$$


We put

$$
U=f^{-1}\left(O_{1 / 4 n} \overline{f(C)}\right) .
$$

Then the set $U$ is a neighborhood of the set $C$ in the space $X$. Therefore,

$$
(A \backslash U) \cap(B \backslash U)=\varnothing
$$

Consequently, there eixsts a function $\varphi: X \rightarrow[0,1]$ such that

$$
\varphi(A \backslash U)=0, \varphi(B \backslash U)=1 .
$$

We will define a mapping $g: X \rightarrow I^{\omega}$ by the equalities

$$
\begin{aligned}
& g_{i}(x)=f_{i}(x) \text { for } i \leqq k, \quad g_{k+1}(x)=\varphi(x), \\
& g_{i}(x)=0 \text { for } i>k+1 .
\end{aligned}
$$

where $g_{i}$ (respectively $f_{i}$ ) is $i$-coordinate of $g$ (respectively $f$ ). Inequality (12) follows from (11), (15). Let us show that

$$
\overline{g(A)} \cap \overline{g(B)} \backslash O_{1 / n} \overline{g(C)}=\varnothing \text {. }
$$

We assume on the contrary. Then there exist two points $a \in A$ and $b \in B$ such that

$$
\delta\left(g(a), g(\overline{C)}) \geqq \frac{1}{n}, \quad \delta(g(b), \overline{g(C)}) \geqq \frac{1}{n}, \quad \delta(g(a), g(b))<2^{-(k+1)}\right.
$$

where $\delta$ is a metric in $I^{\omega}$. From condition (11), (15) it follows that

$$
\delta(\overline{g(A \backslash U)}, \overline{g(B \backslash U)}) \geqq 2^{-(k+1)} .
$$

Consequently

$$
\text { either } g(a) \notin g \overline{(A \backslash U)} \quad \text { or } \quad g(b) \notin g \overline{(B \backslash U)} \text {. }
$$

Let, for example $g(a) \notin \overline{g(A \backslash U)}$, then obviously $a \notin(A \backslash U)$ and, consequently $a \in U$. From condition (13) it follows that

$$
\delta(f(a), \overline{f(C)})<\frac{1}{4 n} .
$$

Consequently, for some point $c \in C$

$$
\delta(f(a), f(c))<\frac{1}{4 n} .
$$

By virtue of (12), (18)

$$
\begin{aligned}
\delta(g(a), \overline{g(C)}) & \leqq \delta(g(a), g(c)) \\
& \leqq \delta(g(a), f(a))+\delta(f(a), f(c))+\delta(f(c), g(c)) \\
& <\frac{3}{4 n}<\frac{1}{n}
\end{aligned}
$$


which contradicts the condition (17). Consequently equality (16) holds and $g \in \phi_{n}$. We have proved that the set $\phi_{n}$ is everywhere dense in $C\left(X, I^{\omega}\right)$. By Lemma $1.1 \phi_{n}$ is open in $C\left(X, I^{\omega}\right)$. Since, obviously

$$
\phi=\bigcap_{n=1}^{\infty} \phi_{n}
$$

then by Baire's theorem $\phi$ is an everywhere dense $G_{j}$-set.

Let $X$ be a space, then we denote by $G_{X}$ the set of all subsets $A \subset C\left(X, I^{\omega}\right)$ such that $A$ contains an everywhere dense $G_{i}$-set in $C\left(X, I^{\omega}\right)$. We note that from Baire's theorem it follows that

$$
\text { if } A_{i} \in G_{X}(i=1,2, \cdots) \text {, then } \bigcap_{i=1}^{\infty} A_{i} \in G_{X} \text {. }
$$

In what follows we shall use the assertion (see [5], Hurewicz and Wallman).

If $X$ is a separable space, then the set of all homeomorphisms $f: X \rightarrow I^{\omega}$ contains an everywhere dense $G_{i}$-set in $C\left(X, I^{\omega}\right)$.

The following two lemmas can be proved by well known standard methods. However we shall prove them for the completeness.

We remind the reader that $g: X \rightarrow Y$ is an $\varepsilon$-mapping if diameter $g^{-1}(y)<\varepsilon$ for any point $y \in Y$.

LEMMA 1.3. Let $K \subset X$ be a compactum in a space $X$, then the set of all mappings $f: X \rightarrow I^{\omega}$ such that the restriction of $f$ to $K$ is a homeomorphism is an everywhere dense $G_{\delta}$-set in $C\left(X, I^{\omega}\right)$.

Proof. Let $\phi_{\varepsilon}$ be a set of all mappings $f: X \rightarrow I^{\omega}$ such that the restriction $f_{K}$ of a mapping $f$ to $K$ is an $\varepsilon$-mapping, and $\psi_{\varepsilon}$ be a set of all $\varepsilon$-mappings $g: K \rightarrow I^{\omega}$. Then $\psi_{\varepsilon}$ is open and everywhere dense in $C\left(K, I^{\omega}\right)$, see [5]. Consequently $\phi_{\varepsilon}$ is open in $C\left(X, I^{\omega}\right)$. Let us show that $\phi_{\varepsilon}$ is everywhere dense. Let $f: X \rightarrow I^{\omega}$ be a mapping and $\delta>0$. Since $\psi_{\varepsilon}$ is everywhere dense there exists an $\varepsilon$-mapping $g: K \rightarrow I^{\omega}$ such that

$$
d\left(f_{K}, g\right) \leqq \frac{\delta}{2}
$$

Since $I^{\omega} \in A R$ there is an extension $\bar{g}: X \rightarrow I^{\omega}$ of a mapping $g$. We put 


$$
h(x)=\left\{\begin{array}{l}
\bar{g}(x) \text { for }|(f-\bar{g})(x)| \leqq \frac{\delta}{2} \\
f(x)+\frac{\delta}{2} \frac{(\bar{g}-f)(x)}{|(\bar{g}-f)(x)|} \text { for }|f-\bar{g}|>\frac{\delta}{2} .
\end{array}\right.
$$

Then, obviously $d(h, f) \leqq \delta / 2<\delta$ and since $g(x)=(x)$ for $x \in K$ we have $h \in \phi_{\varepsilon}$. Therefore, the set $\phi_{\varepsilon}$ is open and everywhere dense in $C\left(X, I^{\omega}\right)$. Our lemma now follows from (19) and the equality

$$
\phi=\bigcap_{n=1}^{\infty} \phi_{1 / n} \text {. }
$$

LEMma 1.4. Let $A$ be a closed subset of a space $X$ and $\operatorname{dim} A \leqq$ $n$, then the set $\phi$ of all mappings $f: X \rightarrow I^{\omega}$ such that

$$
\operatorname{dim} \overline{f(A)} \leqq n
$$

is an everywhere dense $G_{\dot{o}}$-set in $C\left(X, I^{\omega}\right)$.

Proof. Let $K_{\varepsilon}$ be a set of all mappings $f: X \rightarrow I^{\omega}$ such that

$$
\left.d_{n+1}(\overline{f(A})\right)<\varepsilon
$$

where $\left.d_{n+1}(\overline{f(A})\right)$ is an $(n+1)$-coefficient of Urysohn, i.e., the inf of $\varepsilon>0$ such that there is a covering of $\overline{f(A)}$ with open sets of diameter $<\varepsilon$ and of order $\leqq n+1$.

Then, clearly

$$
\phi=\bigcap_{n=1}^{\infty} K_{1 / n} .
$$

Therefore by virtue of (19) it is sufficient to prove that $K_{1 / n}$ is open and everywher dense set. Let $f \in K_{1 / n}$ then there exists a finite collection of open in $I^{\omega}$ sets $V=\left\{V_{1}, \cdots, V_{s}\right\}$ such that

$$
\overline{f(A)} \subset \bigcup_{i=1}^{s} V_{i}, \operatorname{diam} V_{i} \leqq \frac{1}{n}, \text { order } \quad V \leqq n+1 .
$$

It is evident that the set 0 of all mappings $f: X \rightarrow I^{\omega}$ satisfying the condition (21) is a neighborhood of $f$ in $C\left(X, I^{\omega}\right)$. Thus, $K_{1 / n}$ is open. Let us prove that $K_{1 / n}$ is everywhere dense. Let $g: X \rightarrow I^{\omega}$ be a mapping. Since $\operatorname{dim} A \leqq n$ we can construct by Kuratowski method [8] a mapping $f: X \rightarrow I^{\omega}$, such that

$$
d_{X}(f, g)<\varepsilon
$$

and $f(A)$ is contained in $n$-dimensional polyhedra. Therefore $\operatorname{dim} \overline{f(A)} \leqq$ $n$ and $d_{n+1}\left(\overline{f(A))}=0\right.$. Consequently, $f \in K_{1 / p}$. Thus the set $K_{1 / p}$ is everywhere dense in $C\left(X, I^{\omega}\right)$. 
2. Further lemmas for the compactification theorem.

Definition 2.1. A space $X$ is called weakly infinite dimensional if for any countable sequence of pairs $\left\{F_{i}, G_{i}\right\}$ of closed sets in $X$ $(i=1,2, \cdots), F_{i} \cap G_{i}=\varnothing$, there exists for every $i$ a partition $C_{i}$ between $F_{i}$ and $G_{i}$ such that

$$
\bigcap_{i=1}^{k} C_{i}=\varnothing,
$$

for some $k=1,2, \cdots$.

Every finite dimensional space is clearly weakly infinite dimensional.

Definition 2.2. A countable system of open sets $U_{n}, n=1,2, \cdots$, in a space $X$ is called convergent if for any discrete in $X$ sequence of points $\left\{x_{i}\right\}, i=1,2, \cdots$, there exist numbers $p$ and $n, p, n=$ $1,2, \cdots$ such that $x_{i} \in U_{n}$ for $i \geqq p$.

If the system $\left\{U_{n}\right\}$ is convergent then the set $X \backslash \bigcup_{n=1}^{\infty} U_{n}$ is called the limit of this system. Let a space $X$ be weakly infinite dimensional and

$$
\begin{aligned}
U_{n}= & \left\{x: x \in X, \text { there exists a neighborhood } O_{x} \ni x\right. \\
& \text { such that } \left.\operatorname{dim} O_{x} \leqq n\right\} .
\end{aligned}
$$

Then we have, see [19], Sklyarenko:

(S1) The system of sets $\left\{U_{n}\right\}$ is convergent and has a compact limit.

Corollary 2.1. If a space $X$ is infinite dimensional and weakly infinite dimensional then

(i) the set $P(X)$ is a nonempty compactum.

(ii) $\operatorname{Ind}(X \backslash O P(X))<\omega_{0}$ for any neighborhood $O P(X)$ of a compactum $P(X)$.

Proof. The compactness of $P(X)$ follows the equality

$$
P(X)=X \mid \bigcup_{n=1}^{\infty} U_{n}
$$

and Theorem (S1). Let $P(X)=\varnothing$. Since $X$ is infinite dimensional, there exists a sequence of points $x_{i}$ in $X$ such that

$$
x_{i} \in U_{n_{i}} \backslash \cup\left\{U_{j}: j<n_{i}\right\}, \quad n_{i+1}>n_{i}, n_{i}=1,2, \cdots .
$$

From condition (3) it follows that a sequence $\left\{x_{i}\right\}$ is discrete in $X$ but by Theorem (S1) the system $\left\{U_{n}\right\}$ is convergent. We obtain the contradiction. Consequently $P(X) \neq \varnothing$. 
If condition (ii) does not hold then there exists a sequence of points $\left\{x_{i}\right\}$ with property (3) and such that $x_{i} \in X \backslash O P(X)$, consequently a sequence $\left\{x_{i}\right\}$ is discrete in $X$ and we again obtain the contradiction. Therefore the property (ii) holds.

We shall use the following theorem, see [21]; Smirnov.

(SM1) If the space $X$ has dimension Ind $X$ then $X$ is weakly infinite dimensional.

Lemma 2.1. If sets $A, B$ are closed in $X=A \cup B$ and the set $B$ is finite dimensional, $\alpha \geqq \omega_{0}$ and

$$
\text { Ind } A<\alpha \quad \text { (ind } A<\alpha \text { ) }
$$

then

$$
\operatorname{Ind}(A \cup B)<\alpha \quad(\operatorname{ind}(A \cup B)<\alpha) .
$$

This lemma directly follows from [10] (Levshenko), Theorems 1, 1', p. 257.

LEMMA 2.2. If $B$ is a bicompactum (not necessarily metrizable) and $C \subset B \backslash P(B)$ is a closed subset, then

$$
\operatorname{dim} C<\infty \text {. }
$$

Proof. From Defininition 0.4 it follows that every point $x \in C$ has a closed finite dimensional neighborhood $V(x)$. The lemma now follows from the compactness of $C$ and the sum theorem for dimension dim.

LEMMA 2.3. Let $X$ be an arbitrary normal space (not necessarily metrizable) and $(A, B)$ be a pair of two closed disjoint sets in $X$. Let also $P \subset X$ be a closed set and $C$ be a partition in $X$ between $A_{1}=A \cap P$ and $B_{1}=B \cap P$. If the set $C$ has a type $G_{\delta}$ in $X$ then there exists a partition $C_{0}$ in $X$ between $A$ and $B$ such that

$$
C_{0}=C_{1} \cup C_{2} \text {, }
$$

where sets $C_{1}$ and $C_{2}$ are closed in $X, C_{1} \subset C, C_{2} \subset X \backslash O P$ for some neighborhood $O P$ of the set $P$. (We do not suppose here that $A_{1} \neq \varnothing$ and $B_{1} \neq \varnothing$.)

Proof. Since $C$ is a partition between $A_{1}$ and $B_{1}$ there are such open sets $U, V$ of that

$$
U \cap V=\varnothing, X \backslash(U \cup V)=C, A_{1} \subset U, B_{1} \subset V .
$$


Consequently

$$
(A \backslash U) \cap P=(B \backslash V) \cap P=\varnothing .
$$

Since $X$ is a normal space, there exists a closed neighborhood $W$ of the set $P$ such that

$$
W \cap((A \backslash U) \cup(B \backslash V))=\varnothing
$$

and $W$ has a type $G_{i}$. We put

$$
U_{1}=(W \cup A \cup B) \backslash(B \cup(W \backslash U)), V_{1}=(W \cup A \cup B) \backslash(A \cup(W \backslash V)) .
$$

Then sets $U_{1}, V_{1}$ are open in $W \cup A \cup B$. Since $A \cap B=\varnothing$, by virtue of (4), (5), (6)

$$
\begin{aligned}
& U_{1} \cap V_{1}=W \cup A \cup B \backslash A \cup B \cup(W \backslash U) \cup\left(W \backslash V_{l}\right)=\varnothing, \\
& A \subset U_{1}, \quad B \subset V_{1} .
\end{aligned}
$$

Consequently the set

$$
\begin{aligned}
C_{1}=(W \cup A \cup B) \backslash\left(U_{1} \cup V_{1}\right) & =(A \cup(W \backslash V)) \cap(B \cup(W \backslash U)) \\
& =W \cap C \subset C
\end{aligned}
$$

is a partition in $W \cup A \cup B$ between $A$ and $B$. Since the sets $W$ and $C$ are $G_{\dot{\delta}}$, the set $C_{1}=W \cap C$ is also a $G_{\dot{\delta}}$-set. Consequently there exists a continuous function $\varphi: W \cup A \cup B \rightarrow[-1,1]$ such that

$$
\varphi^{-1}(0)=C_{1}, \quad \varphi(A)=-1, \quad \varphi(B)=1 .
$$

Let $\varphi: X \rightarrow[-1,1]$ be any continuous extension of $\varphi$. We put

$$
C_{0}=\varphi^{-1}(0), \quad C_{2}=\overline{C_{0} \mid C_{1}} \text {. }
$$

Then obviously

$$
C_{0}=C_{1} \cup C_{2},
$$

since $C_{0} \backslash C_{1} \subset X \backslash W$ and $W$ is a closed neighborhood of $P$, there exists a neighborhood $O P \subset W$ of the set $P$ such that

$$
C_{0} \backslash C_{1}=C_{2} \subset X \backslash O P \text {. }
$$

Definition 2.3. Let $\mathscr{F}=\left\{F_{i}: i=1,2, \cdots\right\}$ be a countable system of sets in a space $X$ and the set $U \subset X$ be open. Then the system $\mathscr{F}$ is called simple with respect to $U$ if

$$
U=\bigcup_{i=1}^{\infty} F_{i}
$$

$$
F_{i} \cap F_{j}=\varnothing \text { for }|i-j|>1
$$


(9) The system $F$ is locally finite on $U$ and sets $F_{i}$ are closed in $X$.

LEMMA 2.4. Let $B$ be a bicompactum not necessarily metrizable and $f: B \rightarrow K$ be a zero dimensional mapping in a compactum $K{ }^{7}$ If there is a closed set $P \subset B$ and a simple, with respect to $(B \backslash P)$, system $\left\{B_{i}\right\}$ in $B$ such that:

$$
\begin{gathered}
\qquad f(P) \cap f\left(B_{i}\right)=\varnothing \text { for every } i=1,2, \cdots \\
\operatorname{dim} f\left(B_{i}\right)<\infty \\
\text { the restriction of } f \text { to } P \text { is a homeomorphism }
\end{gathered}
$$

and

$$
\text { Ind } K \leqq \alpha
$$

then Ind $B \leqq \alpha$.

Proof. We shall prove this lemma by induction on $\alpha$. If $\alpha<\omega_{0}$ then for any bicompactum $B$ having zero-dimensional mapping in $\alpha$ dimensional compactum $K$ we have

$$
\text { Ind } B \leqq \text { Ind } K \leqq \alpha \text {. }
$$

(See [10], Pasynkov.) Let $\alpha \geqq \omega_{0}$ and for all $\alpha^{\prime}<\alpha$ our lemma is proved. Let $F, G$ be a pair of two disjoint closed sets in $B$. By virtue of (12)

$$
f(F \cap P) \cap f(G \cap P)=\varnothing .
$$

Then by virtue of (13) there exists a partition $D$ between $f(F \cap P)$ and $f(G \cap P)$ such that

$$
\text { Ind } D \leqq \beta<\alpha
$$

We put

$$
C=f^{-1}(D)
$$

Then, since $K$ is metrizable and $D \subset K$ is closed, $D$ is a $G_{\delta}$-set. Consequently, $C$ is also a $G_{\delta}$-set. Moreover the set $C$ is a partition between $F \cap P$ and $G \cap P$ in $B$. By virtue of Lemma 2.3 there exists a partition $C_{0}$ between $F$ and $G$ such that:

$$
C_{0}=C_{1} \cup C_{2}, \quad C_{1} \subset C, C_{2} \subset X \backslash O P
$$

for some closed sets $C_{1}$ and $C_{2}$ and a neighborhood $O P$ of a set $P$.

\footnotetext{
${ }^{6}$ A mapping $f$ is zero dimensional if $\operatorname{dim} f^{-1}(x) \leqq 0$ for any point $x$ in image $f$.

7 In our terminology a compactum is a metrizable bicompactum.
} 
We note that if $B^{\prime} \subset B$ is a closed set, $B_{i}^{\prime}=B_{i} \cap B^{\prime}, P^{\prime}=P \cap B$, $K^{\prime}=f\left(B^{\prime}\right)$ then all conditions of Lemma 2.4 are fulfilled for bicompactum $B^{\prime}$ (we have only to change notation). Consequently, if Ind $f\left(B^{\prime}\right)<\alpha$ then by inductive assumption Ind $B^{\prime} \leqq$ Ind $f\left(B^{\prime}\right)<\alpha$. Therefore for proving inequality

$$
\text { Ind } C_{0}<\alpha
$$

we have only to prove that

$$
\text { Ind } f\left(C_{2}\right)<\infty \text {. }
$$

Indeed, as it was mentioned above we have only to prove that

$$
\text { Ind } f\left(C_{0}\right)=\text { Ind } f\left(C_{1}\right) \cup f\left(C_{2}\right)<\alpha .
$$

By virtue of (14), (15), (16) we obtain the inequality

$$
\text { Ind } f\left(C_{1}\right) \leqq \text { Ind } D \leqq \beta<\alpha .
$$

By Lemma 2.1 and (18), (19)

$$
\text { Ind }\left(f\left(C_{1}\right) \cup f\left(C_{2}\right)\right)<\alpha .
$$

Let us prove inequality (18). By conditions of the lemma we have

$$
B \backslash P=\bigcup_{i=1}^{\infty} B_{i}
$$

and the system $\left\{B_{i}, i=1,2, \cdots\right\}$ is locally finite on $B \backslash P$. Since $C_{2} \subset$ $B \backslash P$ and $C_{2}$ is a subbicompactum of $B$ then for some finite collection $B_{i_{1}}, \cdots, B_{i_{k}}$ we have

$$
C_{2} \subset \bigcup_{s=1}^{k} B_{i_{s}} .
$$

Consequently, by virtue of (11)

$$
\operatorname{dim} f\left(C_{2}\right) \leqq \max \left\{\operatorname{dim} f\left(B_{i_{s}}\right): s=1, \cdots, k\right\}<\infty .
$$

Since $f\left(C_{2}\right) \subset K$ and $K$ is a metrizable space, $\operatorname{dim} f\left(C_{2}\right)=\operatorname{Ind} f\left(C_{2}\right)$. Thus inequality (18) and consequently (17) holds. Therefore Ind $B \leqq \alpha$.

Lemma 2.5. If for any two closed disjoint sets $F, G$ in weakly infinite dimensional space $Z$ such that

$$
F \cup G \subseteq P(Z)
$$

there exists a partition $C$ having the dimension Ind $C<\alpha\left(\alpha \geqq \omega_{0}\right)$ then Ind $Z \leqq \alpha$. 
Proof. Let $(A, B)$ be an arbitrary pair of disjoint closed sets in $Z$. If either $A \cap P(Z)=\varnothing$ or $B \cap P(Z)=\varnothing$ then either the set $A$ or $B$ belongs to the set $Z \backslash O P(Z)$ for some neighborhood $O P(Z)$ of the set $P(Z)$. By virtue of Corollary 2.1 there exists a finite dimensional partition $C$ between $A$ and $B$; consequently

$$
\text { Ind } C<\omega_{0} \leqq \alpha .
$$

Let $A_{1}=A \cap P(Z) \neq \varnothing$ and $B_{1}=B \cap P(Z) \neq \varnothing$. By the given condition there exists a partition $C$ between $A_{1}$ and $B_{1}$ such that

$$
\text { Ind } C<\alpha \text {. }
$$

Since $Z$ is metrizable, $C$ is a $G_{i}$-set and by virtue of Lemma 2.3 there exists a partition $C_{0}$ between $A$ and $B$ such that

$$
C_{1} \subset C, \quad C_{2} \subset X \backslash O P, \quad C_{0}=C_{1} \cup C_{2}
$$

for some closed sets $C_{1}, C_{2}$ and a neighborhood $O P$ of the set $P$. By virtue of Corollary 2.1

$$
\text { Ind } C_{2}<\infty \text {. }
$$

From (20), (21) it follows that

$$
\text { Ind } C_{1}<\alpha \text {. }
$$

The inequality Ind $C_{0}<\alpha$ now follows from Lemma 2.1 and (21).

LEMMA 2.6. If for any point $p \in P(Z)$ in a weakly infinite dimensional space $Z$ and for any closed set $F \subset P(Z), F \nexists p$, there exists a partition $C$ in $Z$ such that

$$
\text { ind } C<\alpha\left(\alpha \geqq \omega_{0}\right)
$$

then ind $Z \leqq \alpha$.

The proof is similar to the proof of Lemma 2.5.

Corollary 2.2. For any weakly infinite dimensional space $Z$ the following assertions hold:

(a) Ind $Z \leqq \alpha \Leftrightarrow$ for any closed sets $F, G, F \cup G \subset P(Z), F \cap G=$ $\varnothing$ there is a partition $C$ with Ind $C<\alpha$. $\alpha \geqq \omega_{0}$.

(b) ind $Z \leqq \alpha \Leftrightarrow$ for any closed set $F \subset P(Z)$ and a point $x \in$ $P(Z) \backslash F$ there is a partition $C$ with ind $C<\alpha . \quad\left(\alpha \geqq \omega_{0}\right)$

Corollary 2.3. If $X$ has the dimension Ind $X$ and $Y \subset X$ is weakly infinite dimensional then 


$$
\text { Ind } Y \leqq \text { Ind } X \text {. }
$$

We shall prove this corollary by induction on $\alpha=$ Ind $X$. If $\alpha<\omega_{0}$ our assertion is known. Let $\alpha \geqq \omega_{0}$ and $F, G$ be a pair of two disjoint closed sets in $P(Y)$. Since $Y \subset X$ we have

$$
P(Y) \subset P(X) \text {. }
$$

Since by Corollary 2.1 $P(Y)$ is a compactum, the sets $F, G$ are closed in $P(X)$ and by inductive assumption there exists a partition $C$ between $F$ and $G$ in $X$ such that

$$
\text { Ind } C<\alpha .
$$

Then $C \cap Y$ is a partition between $F$ and $G$ in $Y$ and since $C \cap Y$ is closed in $Y$ it is weakly infinite dimensional space. Consequently by inductive assumption

$$
\operatorname{Ind}(C \cap Y) \leqq \operatorname{Ind} C<\alpha .
$$

Inequality (22) now follows from Corollary 2.2.

LEMMA 2.7. Let $\mathscr{U}=\left\{U_{i}: i=1,2, \cdots\right\}$ be a system of open sets in a space $X, \alpha$ a limit ordinal number $<\omega_{1}$ and

$$
\begin{aligned}
& D\left(U_{i}\right)<\alpha \\
& U=\bigcup_{i=1}^{\infty} U_{i} .
\end{aligned}
$$

Then for any sequence of ordinal numbers $\gamma_{i}$ such that

$$
\gamma_{i+1}>\gamma_{i}, \quad \sup \gamma_{i}=\alpha \quad i=1,2, \cdots
$$

there exists a simple, with respect to $U$, system $\mathscr{F}=\left\{F_{i}\right\}$ such that

$$
D\left(F_{i}\right) \leqq \gamma_{i} .
$$

Proof. We take a system of open sets $\mathscr{V}=\left\{V_{i}\right\}$ such that

$$
\bar{V}_{i} \subset V_{i+1} \subseteq U, \quad \bigcup_{i=1}^{\infty} V_{i}=U \quad(i=1,2, \cdots) .
$$

Since $X$ is metrizable, there exists an open covering $\left\{D_{i}\right\}$ of the set $U$ such that the covering $\left\{\bar{D}_{i}\right\}$ consisting of closures $\bar{D}_{i}$ is a refinement of $\mathscr{V}$ and $\mathscr{U}$. Since coverings $\mathscr{V}$ and $\mathscr{U}$ are countable, we can require a covering $\left\{D_{i}\right\}$ to be countable. Then there exists a closed covering $\mathscr{H}=\left\{H_{i}\right\}$ of $U$ such that

$$
H_{i}=\bar{H}_{i} \subset D_{i} \quad(i=1,2, \cdots) .
$$


By virtue of (25) the sets $H_{i}, \bar{D}_{i}$ are closed in $X$. From condition (23) it follows that

$$
D\left(H_{i}\right) \leqq D\left(D_{i}\right) \leqq D\left(\bar{D}_{i}\right) \leqq \beta_{i}<\alpha .
$$

We can now construct by induction open sets $W_{i}$ such that:

$$
\begin{gathered}
\bigcup_{k=1}^{i} H_{k} \subset W_{i} \subset \bar{W}_{i} \subset \bigcup_{i=1}^{k} D_{i} \subset U \\
\bar{W}_{i} \subset W_{i+1} .
\end{gathered}
$$

By (27), (28) and the sum theorem for union of finite number of closed sets with $D$-dimension [3], Henderson, we have

$$
D\left(\bar{W}_{i}\right) \leqq \max \left\{D\left(\bar{D}_{i}\right): i=1, \cdots, k\right\}=\delta_{i}<\alpha .
$$

Since $\mathscr{H}$ is a covering of $U$, we have, by virture of (28)

$$
\bigcup_{i=1}^{\infty} W_{i}=U
$$

From conditions (24), (30) it follows that there exists such subsequence $\left\{\gamma_{n(i)}\right\} i=1,2, \cdots$ of a sequence $\left\{\gamma_{i}\right\}$ so that

$$
D\left(\bar{W}_{i}\right) \leqq \gamma_{n(i-1)} \quad n(i+1)>n(i)>0, \quad i=1,2, \cdots .
$$

Since the space $X$ is normal, from condition (29), it follows that for any $i$ there exist such open sets

$$
V_{n(i)}, \cdots, V_{n(i+1)} \quad(i=0,1, \cdots)
$$

so that

$$
\begin{gathered}
V_{n(i)}=W_{i}, \quad V_{n(i+1)}=W_{i+1}, \quad V_{i}=\varnothing \quad \text { for } 0 \leqq i<n(1) . \\
V_{n(i)+k} \subset \bar{V}_{n(i)+k} \subset V_{n(i)+k+1} \quad k=0, \cdots, n(i+1)-n(i) .
\end{gathered}
$$

By virtue of (11), (32), (33), (34), (31) we have

$$
\begin{gathered}
D\left(\bar{V}_{j}\right) \leqq D\left(\bar{W}_{i+1}\right) \leqq \gamma_{n(i)} \leqq \gamma_{j} \text { for } n(i) \leqq j \leqq n(i+1) \\
D\left(\bar{V}_{j}\right)=-1 \leqq \gamma_{j} \text { for } 0 \leqq j<n(1) \\
\bar{V}_{i} \subset V_{i+1} \subset U, \bigcup_{i=1}^{\infty} V_{i}=U .
\end{gathered}
$$

We put

$$
F_{i}=\bar{V}_{i} \backslash V_{i-1} \text { for } i>0, \quad F_{0}=\varnothing .
$$

Then for $j>i, V_{i} \cap F_{j}=\varnothing$. Consequently, by virtue of (37) the system $\left\{F_{i}\right\}$ is locally finite. Conditions (7), (8) follow from (37), (38). Inequality $D\left(F_{i}\right) \leqq \gamma_{i}$ follows from (35), (36), (38). 
Corollary 2.4. For any space $X$ there exists a simple with respect to $X \backslash P(X)$ system $\left\{C_{i}\right\}$ such that

$$
\operatorname{dim} C_{i} \leqq i \quad(i=1,2, \cdots) .
$$

Proof. Let $U_{n}$ be the set defined by equality (1), then obviously

$$
\operatorname{dim} U_{n} \leqq n
$$

and by definition

$$
X \backslash P(X)=\bigcup_{n=1}^{\infty} U_{n} .
$$

Hence by Lemma 2.7 there is a simple, with respect to $X \backslash P(X)$, system $\left\{C_{i}\right\}$ such that

$$
\operatorname{dim} C_{i}=D\left(C_{i}\right) \leqq i=\gamma(i) .
$$

LEMmA 2.8. If $D(X) \geqq \omega_{0}$ then $D(X)=\omega_{0}+D(P(X))$.

Proof. If $D(X)=\Delta$ then our lemma is trivial. Let $D(X)=$ $\beta<\Delta, D(P(X))=\alpha$ and the equality

$$
P(X)=\cup\left\{A_{\zeta}: \xi \leqq J(\alpha)\right\}
$$

be the $\alpha-D$-representation of $P(X)$. By virtue of Corollary 2.4, we have the representation

$$
X=P(X) \cup \bigcup_{i=1}^{\infty} C_{i}
$$

for simple, with respect to $X \backslash P(X)$, system $\left\{C_{i}\right\}$ such that property (39) holds. Let us put

$$
B_{\omega_{0}+\xi}=A_{\xi}, \quad B_{i}=C_{i}, \text { for } i<\omega_{0} .
$$

Then clearly the equality

$$
X=\cup\left\{B_{\xi}: \xi \leqq J\left(\omega_{0}+\alpha\right)=\omega_{0}+J(\alpha)\right\}
$$

is a $\left(\omega_{0}+\alpha\right)-D$-representation of $X$. Therefore

$$
\beta \leqq \omega_{0}+\alpha \text {. }
$$

Let the equality

$$
X=\left\{A_{\xi}=\xi \leqq J(\beta)\right\}
$$

be a $\beta$-D-representation of $X$ and $\beta=\omega_{0}+\delta$. Let us put

$$
B_{\xi}=A_{\omega_{0}+\xi}
$$


and

$$
T=\cup\left\{B_{\xi}: \xi \leqq J(\delta)\right\} \text {. }
$$

Then (40) is clearly a $\delta$-D-representation of $T$. From conditions (b), (c), (e) of Definition 0.3 it follows that the set $X \backslash T$ is a union of open finite dimensional sets. Consequently, $P(X) \subset T$ and

$$
D(P(X)) \leqq D(T) \leqq \delta
$$

Hence $\omega_{0}+\delta \geqq \omega_{0}+\alpha$.

3. Standard representations and standard mappings.

DEFINITION 3.1. Let

$$
X=P(X) \cup \bigcup_{i=0}^{\infty} C_{i}
$$

Then equality (1) is called a standard representation of a space $X$ if

(a) The system $\left\{C_{i}\right\}$ is simple with respect to $X \backslash P(X)$. (In particular $C_{i} \cap P(X)=C_{i} \cap C_{j}=\varnothing$ for $|i-j|>1$.)

(b) $\operatorname{dim} C_{i} \leqq n(i)$.

(c) For any $x \in C_{i}, \delta(x, y)<1 / i$ for some $y \in P(X), i>0$, where $\delta$ is a metric on $X$.

LEMMA 3.1. Let $X$ be a weakly infinite dimensional space. Then there exists a standard representation of $X$.

Proof. We put

$$
\begin{aligned}
& C_{i}=\left\{x: \frac{1}{i+2} \leqq \delta(x, P(X)) \leqq \frac{1}{i+1}\right\} \text { for } i \geqq 1, \\
& C_{0}=\left\{x: \delta(x, P(X)) \geqq \frac{1}{2}\right\} .
\end{aligned}
$$

Then, clearly the equality (1) and properties (a), (c) hold. Property (b) follows from Corollary 2.1.

DEFINITION 3.2. Let (1) be a standard representation of $X$ and $f: X \rightarrow I^{\omega}$ be a mapping such that:

(a) $f$ is a homeomorphism on $P(X)$.

(b) $\overline{f\left(C_{i}\right)} \cap f(P(X))=\overline{f\left(C_{j}\right)} \cap \overline{f\left(C_{i}\right)}=\varnothing$ for $|i-j|>1$.

(c) $\operatorname{dim} \overline{f\left(C_{i}\right)} \leqq \operatorname{dim} C_{i} \leqq n_{i}<\infty$.

Then $f$ is called a standard mapping.

Lemma 3.2. Let $f: X \rightarrow I^{\omega}$ be a standard mapping of a weakly 
infinite dimensional and infinite dimensional space $X$. Then

$$
\overline{f(X)}=f(P(X)) \cup \bigcup_{i=1}^{\infty} \overline{f\left(C_{i}\right)} .
$$

(3) the system $\left\{\overline{f\left(C_{i}\right)}\right\}$ is locally finite on $\overline{f(X) \backslash} f(P(X))$.

$$
\begin{gathered}
f^{-1} P \overline{f(X)} \subset P(X) . \\
D \overline{f(X)} \leqq D(X) .
\end{gathered}
$$

Proof. Let us show that

(6) for any sequence $\left\{y_{i}\right\}$, such that $y_{i} \in \overline{f(X)}, y_{i} \notin \overline{f\left(C_{j}\right)}$ for $j<i$ we have

( 7 ) $\lim _{i \rightarrow \infty} y_{k(i)}=a$ for some point $a \in f(P(X))$, and some subsequence $\left\{y_{k(i)}\right\}$ of sequence $\left\{y_{i}\right\}$.

Indeed, we can take for any $i$ a point $f\left(x_{i}\right)$ such that

$$
\delta\left(f\left(x_{i}\right), y_{i}\right)<\frac{1}{i}, \quad f\left(x_{i}\right) \notin \overline{f\left(C_{j}\right)} \text { for } j<i .
$$

Consequently $x_{i} \in P(x) \cup \cup\left\{C_{j}: j \geqq i\right\}$ and by condition (c) of Definition 3.1 we have

$$
\delta\left(x_{i}, P(X)\right)<1 / i
$$

Since $X$ is weakly infinite dimensional, $P(X)$ is compact and from (9) it follows that

$\lim _{i=\infty} x_{n(i)}=b$ for some point $b \in P(X)$ and some subsequence
$\left\{x_{n(i)}\right\}$ of sequence $\left\{x_{i}\right\}$. Let $f(b)=a$, then property (7) follows from (8), (10). Let us prove (2). Let $x \in \overline{f(X)} \backslash f(X), x \notin \overline{f\left(C_{i}\right)}$ for any $i$, and $x \notin f(P(X))$, then clearly we can construct a sequence $\left\{y_{i}\right\}$ such that

$$
\lim _{i \rightarrow \infty} y_{i}=x \text { and } y_{i} \notin \overline{f\left(C_{j}\right)} \text { for } j<i .
$$

From condition (7) it follows that $x \in f(P(X))$ and we obtain the contradiction. Hence property (2) holds. Similarly we can prove (3). Indeed, if $x \in \overline{f(X)} \backslash f(P(X))$ and any neighborhood $O x$ of $x$ contains points of infinitely many sets $\overline{f\left(C_{i}\right)}$, we can construct a sequence $\left\{y_{i}\right\}$ satisfying (6) and such that

$$
\lim _{i \rightarrow \infty} y_{i}=x \text {. }
$$

By virtue of (7) $x \in f(P(X))$ and we again get the contradiction. 
Therefore the system $\left\{\overline{f\left(C_{i}\right)}\right\}$ is locally finite on $\overline{f(X) \backslash f}(P(X))$. Let us prove (4). Since $P(X)$ is compact, the set $f(P(X))$ is closed and the set $\overline{f(X)} \backslash f(P(X)$ is open in $\overline{f(X)}$. By virtue of condition (2) and (b) of Definition 3.2 we have

$$
\begin{aligned}
& \overline{f(X)} \backslash f(P(X))=\bigcup_{i=1}^{\infty} \overline{f\left(C_{i}\right)} \\
& f^{-1} f(P(X))=P(X) .
\end{aligned}
$$

From conditions (3) and (c) of Definition 3.2 it follows that

$$
P \overline{f(X)} \subset f(P(X)) \text {. }
$$

Consequently, condition (4) follows from (11), (12). Property (5) follows from (12). Indeed, by Lemma 2.8

$$
\begin{aligned}
D(\overline{f(X)}) & =\omega_{0}+D(\overline{P(X)}) \leqq \omega_{0}+D f(P(X)) \\
& =\omega_{0}+D(P(X))=D(X) .
\end{aligned}
$$

We used here the equality $D f(P(X))=D(P(X)$ which follows from condition (a) of Definition 3.2.

Lemma 3.3. Let $U$ be an open set in some closed subset $A$ of a space $X$ and $\phi$ the set of all mappings $f: X \rightarrow I^{\omega}$ such that

$$
f(U) \cap \overline{f(A \backslash} \bar{U})=\varnothing
$$

then

$$
\phi \in G_{X}
$$

Proof. Let $\left\{F_{i}: i=1,2, \cdots\right\}$ be a collection of closed sets in a space $X$ such that $\bigcup_{i=1}^{\infty} F_{i}=U$. Then by virtue of Lemma 1.2 and (19) $\S 1$ the set is of all mappings $f: X \rightarrow I^{\omega}$ such that

$$
\overline{f\left(\overline{F_{i}}\right)} \cap \overline{f(A \backslash U)}=\varnothing
$$

belongs to $G_{X}$. Since clearly $\phi \supset \psi$, we have also $\phi \in G_{X}$.

LEMMA 3.4. Let $X$ be a weakly infinite dimensional closed subset of a space $Y$ and $\phi$ be a set of all mappings $f: Y \rightarrow I^{\omega}$ such that $f$ $i s$ standard on $X$. Then $\phi \in G_{Y}$.

Proof. By Lemma 3.1 there exists a standard representation (1) of a space $X$. Since the system $\left\{C_{i}\right\}$ is countable, our lemma follows from Lemmas 1.2, 1.3, 1.4 and (19) $\S 1$.

Lemma 3.5. Let $U$ be an open set in a space $X$ and $V=\operatorname{Int} \bar{U}$ 
(where $\operatorname{Int} \bar{U}$ is an interior of $\bar{U}$ ). Then

$$
F r V \subset F r U, \quad V=\operatorname{Int} \bar{V}, \quad U \subset V
$$

and the set $X \backslash(F r V \cup V)$ is everywhere dense in $X \backslash V$. The lemma is trivial.

An open set $V$ such that $V=\operatorname{Int} \bar{V}$ is called canonic.

LEMMA 3.6. Let Ind $X=\alpha$, ind $X=\beta\left(\alpha \geqq \beta \geqq \omega_{0}\right)$ and $\lambda(\mu)=$ $\left\{U_{r}^{\lambda}: \gamma \in \Gamma\right\} \mu=a, b$ be two system of open canonic in $X$ sets such that for all $\gamma \in \Gamma$ :

(k1) Ind $\operatorname{Fr} U_{r}^{a}<\alpha$

(k2) ind $F r U_{r}^{b}<\beta$ $P(X)$.

(k3) $\lambda(a)$ forms a large base in $P(X)^{8}$ and $\lambda(b)$ forms a base in Then if $f: X \rightarrow I^{\omega}$ is a standard mapping such that:

(k4) Ind $F r U_{\gamma}^{\alpha} \geqq$ Ind $\overline{f\left(F r U_{r}^{a}\right)}$

(k5) ind $F r U_{r}^{b} \geqq$ ind $\overline{f\left(F r U_{r}^{b}\right)}$

(k6) $\overline{f\left(\bar{U}_{r}^{\mu}\right)} \cap \overline{f\left(X \backslash U_{r}^{\mu}\right)}=\overline{f\left(F r U_{r}^{\mu}\right)}$

(k7) If $\bar{U}_{\gamma_{1}}^{\mu} \subset U_{r_{2}}^{\mu}$ then $\left.\overline{f\left(\bar{U}_{\gamma_{1}}^{\mu}\right)} \cap \overline{f\left(X \backslash U_{r_{2}}^{\mu}\right.}\right)=\varnothing$

(k8) $\left.\overline{f\left(\overline{F r} U_{\gamma}^{\mu}\right.}\right) \cap f\left(X \backslash F r U_{r}^{\mu}\right)=\varnothing$.

For all $\gamma \in \Gamma$ and $\mu=a, b$ then

(a) Ind $\overline{f(X)} \leqq$ Ind $X$

(b) ind $\overline{f(X)} \leqq$ ind $X$

(c) $D \overline{f(X)} \leqq D(X)$.

Moreover, for proving condition (a) it is sufficient to assume that conditions $\mathrm{k} 1, \mathrm{k} 3, \mathrm{k} 4, \mathrm{k} 6, \mathrm{k} 7, \mathrm{k} 8$ hold and for proving condition (b) it is sufficient to assume that conditions $\mathrm{k} 2, \mathrm{k} 3, \mathrm{k} 5, \mathrm{k} 6, \mathrm{k} 7, \mathrm{k} 8$ hold, and condition (c) follows from the standardness of mapping $f$.

Proof. Condition (c) follows from Lemma 3.2, property (5). Let us prove (a). Let $F, G$ be a pair of disjoint closed sets in $\overline{P f(X)}$. We put

$$
F_{1}=f^{-1}(F), \quad G_{1}=f^{-1}(G) .
$$

Since $f$ is a standard mapping, by Lemma 3.2(4) we have

$$
F_{1} \cup F_{2} \subseteq P(X)
$$

and clearly

$$
F_{1} \cap F_{2}=\varnothing \text {. }
$$

8 This means that for any pair $(F, G)$ of closed disjoint sets in $(X)$ there is a set $U_{r}^{a}$, open in $X$, such that $F \subset U_{\gamma}^{a} \subset X / G$. 
By virtue of $\mathrm{k} 1, \mathrm{k} 3$ there exist such sets $U_{\gamma_{1}}^{a}, U_{\gamma_{2}}^{\alpha}, U_{\gamma_{3}}^{a} \in \lambda(a)$ so that

$$
\begin{gathered}
F_{1} \subset U_{\gamma_{1}}^{a} \subset \bar{U}_{\gamma_{1}}^{a} \subset U_{\gamma_{1}}^{a} \subset \bar{U}_{\gamma_{2}}^{a} \subset U_{r_{3}}^{a} \subset X \backslash G_{1}, \\
\text { Ind } F r U_{\gamma_{2}}^{a}<\alpha .
\end{gathered}
$$

We put

$$
\left.U=U_{\gamma_{2}}^{a}, V=\operatorname{Int} \overline{f\left(\bar{U}_{\gamma_{2}}^{a}\right)}=\overline{f\left(\bar{U}_{\gamma_{2}}^{a}\right)} \backslash \overline{F r f\left(\bar{U}_{\gamma_{2}}^{a}\right.}\right), A=\overline{\overline{f(X)} \backslash \overline{f\left(\bar{U}_{\gamma_{2}}^{a}\right)}} .
$$

Since $f$ is a continuous mapping,

$$
\overline{f(\bar{U})}=\overline{f(\bar{U})} \text {. }
$$

Let us show that

$$
A=\overline{f(X \backslash U)} .
$$

By virtue of $(\mathrm{k} 6),(\mathrm{k} 8),(18)$

$$
\overline{f(U)} \cap \overline{f(X \backslash U)} \cap f(X \backslash F r U)=\varnothing .
$$

Since $U$ is canonic set, from Lemma 3.5 it follows that the set $\overline{f(X \backslash U)} \cap f(X \backslash F r U)$ is everywhere dense in $\overline{f(X \backslash U)}$, consequently

$$
\overline{f(X \backslash U)}=\overline{\overline{f(X \backslash U) \cap f(X \backslash F r U})} \text {. }
$$

From (18), (20), (21) it follows that

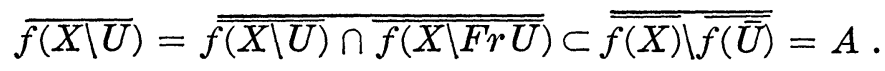

Since

$$
\overline{f(X)} \backslash \overline{f(U)}=(\overline{f(X \backslash U}) \cup \overline{f(U)}) \backslash \overline{f(U)} \subset \overline{f(X \backslash U})
$$

and the set $\overline{f(X \backslash U)}$ is closed, we have

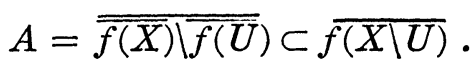

The condition (19) follows from (22), (23). By virtue of (17), (18), (19) we have

$$
V=\overline{f(U)} \backslash(\overline{f(U)} \cap(\overline{\overline{f(X)} \backslash \overline{f(U})}))=\overline{f(\bar{U})} \backslash(\overline{f(U)} \cap \overline{f(X \backslash U)}) .
$$

From conditions (13), (15), (17), k7 it follows that

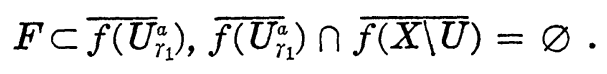

Consequently,

$$
F \subset V \text {. }
$$

On the other hand, by virtue of $\mathrm{k} 7,(15),(24),(13)$ 


$$
\left.V \subseteq \overline{f(U)}, \overline{f(U)} \cap \overline{f\left(X \backslash U_{r_{3}}^{a}\right)}=\varnothing, \quad G \subset \overline{f\left(X \backslash U_{r_{3}}^{a}\right.}\right)
$$

Consequently

$$
\bar{V} \subset \overline{f(X)} \mid G .
$$

Since the set $V$ is open (condition 17), it follows from (25) and (26) that $F r V$ is a partition between $F$ and $G$ in $\overline{f(X)}$. Since by (24) $\bar{V} \subset \overline{f(U)}$ and $V$ is open, we have

$$
\operatorname{Fr} V=\bar{V} \backslash V \subset \overline{f(U)} \backslash V=\overline{f(U)} \cap \overline{f(X \backslash U)} .
$$

But by virtue of (19), (17)

$$
\operatorname{Fr} \overline{f(U)}=\overline{f(U)} \cap \overline{\overline{f(X) \backslash \overline{f(U}})})=\overline{f(U)} \cap \overline{f(X \backslash U}) .
$$

From (k6), (27), (28) it follows that

$$
\begin{aligned}
& \overline{f(F r U)}=F r \overline{f(U)} \supset F r V \\
& \overline{f(F r U)}=\operatorname{Fr} \overline{f(U)} \supset F r V .
\end{aligned}
$$

By virtue of $(\mathrm{k} 4),(16),(17)$

$$
\text { Ind } \overline{f(F r U)} \leqq \text { Ind } F r U<\alpha .
$$

Consequently Ind $F r V<\alpha$. Inequality (a) now follows from Corollary 2.2(a). Similarly one can prove inequality (b).

4. Proof of the compactification theorem. First we shall prove some general theorems.

TheOREM 4.1. Let $X$ be a closed subset of a space $Y$ and dimension Ind $X$ exists. Let $\phi$ be the set of all standard on $X$ mappings $f: Y \rightarrow I^{\omega}$ such that

$$
\text { Ind } \begin{aligned}
\overline{f(X)} & \leqq \text { Ind } X \\
D \overline{f(X)} & \leqq D(X)
\end{aligned}
$$

Then $\phi \in G_{Y}$.

LeMma 4.1. Let $X$ be a closed subset of a space $Y$ and dimension Ind $X$ exists. (We note that by virtue of (2) (introduction) the dimension ind $X$ also exists.) Let Ind $X=\alpha$, ind $X=\beta$ and $\beta \geqq \omega_{0}$. Then there exist two countable systems $\lambda(\mu)=\left\{U_{\gamma}^{\mu}: \gamma \in \Gamma\right\} \mu=a, b$ of open in $X$ canonic sets such that for all $\gamma \in \Gamma$ conditions $\mathrm{k} 1, \mathrm{k} 2, \mathrm{k} 3$ of Lemma 3.6 are fulfilled.

Proof. The existence of systems $\lambda(\mu)$ with properties k1, k2, k3 
is evident. Since Ind $X$ exists by (SM1) $\S 2$ and Corollary 2.1, $P(X)$ is compact. Consequently we can consider systems $\lambda(\mu)$ to be countable. Indeed, from any base (large base) of compact metric space we can select a countable subsystem which is also a base (large base). By virtue of Lemma 3.5 all elements of these bases we can consider to be canonic.

LEMMA 4.2. Let in Lemma $4.1 \phi_{1}$ be the set of all mappings $f: Y \rightarrow I^{\omega}$ such that $f$ is standard on $X$ and conditions $\mathrm{k} 6, \mathrm{k} 7, \mathrm{k} 8$ of Lemma 3.6 are fulfilled. Then $\phi_{1} \in G_{Y}$.

Proof. Let $\phi_{i}$ be the set of all mappings $f: Y \rightarrow I^{\omega}$ such that the condition $k_{i}(i=6,7,8)$ of Lemma 3.6 holds. Since

$$
\operatorname{Fr} U_{\gamma}^{\mu}=\bar{U}_{\gamma}^{\mu} \cap X \backslash U_{\gamma}^{\mu}
$$

and the systems $\lambda(\mu)$ are countable, from Lemma 1.2 and (19) $\S 1$ it follows that

$$
\phi_{\theta} \in G_{X}
$$

Analogously $\phi_{7} \in G_{X}$. Inclusion $\phi_{8} \in G_{X}$ follows from (19) $\S 1$ and Lemma 3.3. Let $\psi$ be the set of all mappings $f: Y \rightarrow I^{\omega}$ such that $f$ is standard on $X$. Then $\psi \in G_{X}$ by Lemma 3.4. Since obviously

$$
\dot{\phi}_{1}=\psi \cap \phi_{6} \cap \phi_{7} \cap \phi_{8},
$$

our lemma follows from (19) $§ 1$.

Proof of Theorem 4.1. We shall prove the theorem by induction on $\alpha=$ Ind $X$. Let $\alpha<\omega_{0}$ then by virtue of (3) (introduction)

$$
\text { Ind } X=\operatorname{dim} X=D(X)
$$

and our theorem follows from Lemma 1.4. Let $\alpha \geqq \omega_{0}$ then by Lemma 4.1 there exists a countable system $\lambda(a)$ of open canonic in $X$ sets satisfying conditions $\mathrm{k} 1, \mathrm{k} 3$ of Lemma 3.6. Let $\psi$ be the set of all mappings $f: Y \rightarrow I^{\omega}$ such that the condition (k4) is fulfilled. Then from inductive assumption and (19) $\S 1$ it follows that

$$
\psi \in G_{Y} \text {. }
$$

Consequently,

$$
\dot{\phi}_{0}=\psi \cap \phi_{1} \in G_{Y}
$$

where $\phi_{1}$ is the set defined in Lemma 4.2. Let $f \in \phi_{0}$, then $f$ is standard on $A$ mapping. Morerver the conditions (k1), (k3), (k4), (k6), (k7), (k8) are fulfilled. Consequently, by Lemma $3.6 f \in \phi$, where $\phi$ 
is defined in Theorem 4.1. Hence $\phi_{0} \subset \phi$. Consequently $\phi \in G_{Y}$.

THEOREM 4.2. Let $A$ be a closed subset of a space $X$ and dimension Ind $A$ exists. Let $A$ be strongly metrizable ${ }^{9}$ and $\phi$ be the set of all standard on $A$ mappings $f: X \rightarrow I^{\omega}$ such that

$$
\begin{aligned}
\text { Ind } \overline{f(A)} & \leqq \operatorname{Ind}(A) \\
D \overline{f(A)} & \leqq D(A) \\
\text { ind } \overline{f(A)} & \leqq \text { ind } A .
\end{aligned}
$$

Then $\phi \subset G_{X}$.

Proof. We shall prove the theorem by induction on $\beta=$ ind $A$. By virtue of Theorem 4.1 it is sufficient to prove that the set $\psi$ of all mappings satisfying condition (1) belongs to $G_{X}$. Let $\beta<\omega_{0}$, then

$$
\text { ind } A=\operatorname{Ind} A=\operatorname{dim} A \text {, }
$$

see [23], Zarelua. Consequently, our theorem now follows from Lemma 1.4. Let $\beta \geqq \omega_{0}$. In this case our proof is completely similar to the proof of Theorem 4.1.

CoROLlary 4.1. Let $X$ be a space and $\left\{L_{i}\right\}$ be a countable system of closed subsets such that the dimension Ind $L_{i}$ exists for every $i$. Let $\Phi$ be the set of all mappings $f: X \rightarrow I^{\omega}$ such that

(a) Ind $\overline{f\left(L_{i}\right)} \leqq$ Ind $L_{i} \quad(i=1,2, \cdots)$.

(b) $D \overline{f\left(L_{i}\right)} \leqq D\left(L_{i}\right)$.

(c) if $L_{j}$ is strongly metrizable for some $j$ then ind $\overline{f\left(L_{j}\right)} \leqq$ ind $L_{j}$.

(d) $f$ is a standard mapping on $L_{i}$.

(e) $f^{-1}\left(P \overline{f\left(L_{i}\right)}\right) \subset P\left(L_{i}\right)$

for any $i=1,2, \cdots$. Then $\Phi \in G_{X}$.

Proof. By Lemma 3.2 the condition (e) follows from (d). Our corollary now follows from Theorem 4.1, Theorem 4.2 and (19) $§ 1$.

Proof of Compactification Theorem (1.1). Let $\psi_{0}$ be the set of all homeomorphisms $f: X \rightarrow I^{\omega}$ of separable space $X$ in Hilbert cube. Then, see [5], Hurewicz and Wallman,

$$
\psi_{0} \in G_{X}
$$

Since for every $i$ and $f \in \psi_{0}$

${ }^{\theta}$ The condition of strong metrizability of $X$ is equivalent to the following one:

There exists an imbedding $f: X \rightarrow I^{\omega} \times \Pi_{i=1}^{\infty} B_{i}$, where $I^{\omega} \times \Pi_{i=1}^{\infty} B_{i}$ is a product of Hilbert cube and a countable number of discrete spaces. 


$$
\text { Ind } f\left(L_{i}\right)=\operatorname{Ind} L_{i}, \quad \text { ind } f\left(L_{i}\right)=\text { ind } L_{i}, \quad D\left(f\left(L_{i}\right)\right)=D\left(L_{i}\right)
$$

and $f\left(L_{i}\right) \subset \overline{f\left(L_{i}\right)}$, we have

$$
\text { ind } L_{i} \leqq \text { ind } \overline{f\left(L_{i}\right)}, \quad D\left(L_{i}\right) \leqq D \overline{f\left(L_{i}\right)}
$$

see [3] (introduction). Inequality

$$
\text { Ind } L_{i} \leqq \operatorname{Ind} \overline{f\left(L_{i}\right)}
$$

follows from Corollary 2.3 and SM1 $\S 2$. Let $\phi_{0}=\phi \cap \psi_{0}$, where $\phi$ is defined in Corollary 4.1. Then from Corollary 4.1, (2) and (19) §1 it follows that

$$
\phi_{0} \in G_{X}
$$

Since $f$ is a homeomorphism, we have $f(P(X))=P(f(X)) \subset P \overline{f(X)}$ Inclusion $\overline{P f(X)} \subset f(P(X))$ follows from condition (e) of Corollary 4.1. Consequently condition (d) of the Compactification Theorem also holds. We obtain that $\psi \supset \phi_{0}$, where the set $\psi$ was defined in Theorem 1.1 and, by virtue of (5), $\psi \in G_{X}$. The theorem is proved.

\section{Uniformly zero dimensional mappings.}

THEOREM 5.1. Let $X$ be a space and a fixed countable system of closed sets $L_{i}, i=1,2, \cdots$ such that dimensions Ind $L_{i}$ exist. Then the set \& of all uniformly zero dimensional mappings ${ }^{10} f: X \rightarrow I^{\omega}$ of the space $X$ to the Hilbert cube $I^{\omega}$ such that for each $i$ we have:

(a) Ind $L_{i}=\operatorname{Ind} \overline{f\left(L_{i}\right)}$

(b) $D\left(L_{i}\right)=D \overline{f\left(L_{i}\right)}$

(c) ind $L_{i} \leqq$ ind $\overline{f\left(L_{i}\right)} \leqq \omega_{0}+$ ind $L_{i}$

(d) ind $L_{i}=$ ind $\overline{f\left(L_{i}\right)}$ if $L_{i}$ is strongly metrizable

(e) $f$ is a standard mapping on $L_{i}$ contains an everywhere dense set of type $G_{\delta}$ in the space $C\left(X, I^{\omega}\right)$.

To prove this theorem we need some preliminary lemmas.

LEMMA 5.1. Let $X$ be a bicompactum, not necessarily metrizable (respectively by a metric space) and $f: X \rightarrow Y$ be a zero dimensional mapping (respectively uniformly zero dimensional) in a compactum Y. Then
(a) ind $Y \geqq$ ind $X$
(b) $D(Y) \geqq D(X)$.

10 We recall that $f: X \rightarrow Y$ is uniformly zero dimensional if for any $\varepsilon>0$ there exists $\delta>0$ such that for every set $\mathscr{M} \subset Y$ of diameter $\mathscr{N}<\delta$ the set $f^{-1}(\mathscr{C})$ is a union of a discrete collection of sets, each of them having the diameter $<\varepsilon$. 
Proof. Inequality (a) was proved in [23] (Theorem 1), Zarelua. Let $D(Y)=\alpha$ and

$$
Y=\left\{Y_{r}: \gamma \leqq J(\alpha)\right\}
$$

be an $\alpha$ - $D$-representation of $Y$. Since zero dimensional mappings of bicympacta (respectively uniformly zero dimensional mappings of metric spaces) do not lower dimension Ind; see [17] (respectively [6]), it is easy to see that

$$
X=\cup\left\{Y=f^{-1}\left(Y_{r}\right): \gamma \leqq J(\alpha)\right\}
$$

is an $\alpha-D$-representation of $X$. Consequently, $D(X) \leqq \alpha$.

LEMMA 5.2. Let $X, Y$ be spaces with dimensions Ind $X$, Ind $Y$ and $f: X \rightarrow Y$ be a uniformly zero dimensional standard mapping. Then Ind $Y \geqq$ Ind $X$.

Proof. We shall prove this theorem by induction on Ind $Y$. Let Ind $Y=\alpha$ and $\alpha<\omega_{0}$. Then $\operatorname{dim} Y=D(Y)=\operatorname{Ind} Y$ ((3) introduction) and our lemma follows from Lemma 5.1(b). Let $\alpha \geqq \omega_{0}$ and $F, G$ be a pair of closed disjoint subsets in $X$ such that

$$
F \cup G \subset P(X) \text {. }
$$

By virtue of (a) definition 3.2 we have

$$
f(F) \cap f(G)=\varnothing \text {. }
$$

Since $X$ is weakly infinite dimensional (SM1 $\S 2$ ), $P(X)$ is compact by Corollary 2.1 and consequently sets $f(F), f(G)$ are closed. Let $C$ be a partition between $f(F)$ and $f(G)$ such that

$$
\text { Ind } C<\alpha \text {. }
$$

Then $f^{-1}(C)$ is a partition between $F$ and $G$ in $X$. Since clearly the restriction of any uniformly zero dimensional standard mapping to a closed subset is also zero dimensional standard mapping we have by inductive assumption and (1)

$$
\text { Ind } f^{-1}(C) \leqq \text { Ind } C<\alpha \text {. }
$$

Our lemma now follows from Corollary 2.2(a).

Lemma 5.3. Let $X$ be a space with dimension Ind $X$. Then ind $X \leqq \omega_{0}+$ ind $P(X) .^{11}$

Proof. We will prove this lemma by induction on ind $P(X)$. If

11 We consider that $\omega_{0}+(-1)=\omega_{0}$. 
ind $P(X)=0$, then for any point $x \in P(X)$ and a closed set $F \subset P(X) \backslash\{x\}$ there exists a partition $C$ in $X$ between $\{x\}$ and $F$ such that

$$
C \cap P(X)=\varnothing \text {. }
$$

Then by Theorem SM1 and Corollary 2.1, $C$ is finite dimensional and ind $C<\omega_{0}+$ ind $P(X)$. Therefore ind $X \leqq \omega_{0}+$ ind $P(X)$ by Corollary 2.2(b). If ind $P(X) \geqq 1$ then for any point $x \in P(X)$ and a closed set $F \subset P(X) \backslash\{x\}$ there exists a partition $C$ in $X$ between $\{x\}$ and $F$ such that

$$
\operatorname{ind}(C \cap P(X))<\text { ind } P(X) \text {. }
$$

Since clearly $P(C) \subset C \cap P(X)$ we have by inductive assumption that

$$
\text { ind } C \leqq \omega_{0}+\text { ind } P(C)<\omega_{0}+\text { ind } P(X) \text {. }
$$

Our lemma now follows from Corollary 2.2(b).

Proof of Theorem 5.1. Let $R$ be the set of all uniformly zero dimensional mappings $f: X \rightarrow I^{\omega}$. Then by virtue of [6], Theorem 2.15 , p. 359 and (B), p. 354, Katetov

$$
R \in G_{X} \text {. }
$$

Let $\phi$ be the setdefined in Corollary 4.1. By virtue of this corollary

$$
\phi \in G_{X} .
$$

To prove our theorem it is sufficient to show that

$$
\psi \supset \phi \cap R .
$$

Let $f \in \phi \cap R$. Then (e) is evident and (a), (b), (d) follow from Corollary 4.1 and Lemmas 5.1,5.2. Let us prove (c). Inequality

$$
\text { ind } L_{i} \leqq \operatorname{ind} f\left(L_{i}\right)
$$

follows from Lemma 5.1. By virtue of Lemma 5.3

$$
\text { ind } \overline{f\left(L_{i}\right)} \leqq \omega_{0}+\text { ind } P \overline{f\left(L_{i}\right)} \text {. }
$$

By Corollary 4.1(e) $f^{-1} P f\left(L_{i}\right) \subset P\left(L_{i}\right)$ and since $f$ is a homeomorphism on $P\left(L_{i}\right)$ (see Definition 3.2(a)), we have

$$
\text { ind } P \overline{f\left(L_{i}\right)} \leqq \text { ind } P\left(L_{i}\right) \leqq \text { ind } L_{i} \text {. }
$$

Property (c) now follows from (4), (5), (6). Thus property (3) and Theorem 5.1 are proved.

6. On bicompactifications of metric spaces. 
THeOREM 6.1. For any space $X$ with dimension Ind $X$ there exists a bicompactification $b X \supset X$ such that:

(a) Ind $b X=\operatorname{Ind} X$

(b) $D b X=D X$

(c) ind $X \leqq$ ind $b X \leqq \omega_{0}+$ ind $X$

(d) ind $b X=$ ind $X$ if $X$ is strongly metrizable

(e) weight $b X=$ weight $X$.

Property (a) was also proved in [17], Pasynkov, for normal spaces. We note that for every metric space $X$ there is not a bicompactum $b X$ such that ind $b X=$ ind $X$, Ind $b X=$ Ind $X . \quad$ For example, if ind $X=0$, Ind $X=1$ (see [18], Roy) then for any bicompactum $b X$ with ind $b X=$ 0 we have also Ind $b X=0$.

We recall that a mapping $f: X \rightarrow Y$ is called scattering (see [23], Zarelua) if for any point $x \in X$ and its any neighborhood $U \ni x$ there is a neighborhood $V \ni f(x)$ such that for some open sets $W_{1}, W_{2} \subset X$ we have:

$$
f^{-1}(V)=W_{1} \cup W_{2}, \quad W_{1} \cap W_{2}=\varnothing, \quad x \in W_{1} \subset U .
$$

Every uniformly zero dimensional mapping is obviously scattering.

Proof of Theorem 6.1. Since for a space $X$ with weight $X<\boldsymbol{\aleph}_{0}$ the theorem is trivial, we suppose weight $X \geqq \boldsymbol{\aleph}_{0}$. By virtue of Theorem 5.1 there exists a uniformly zero dimensional mapping $f: X \rightarrow K$ in compact $K \subset I^{\omega}$ such that we put $b X=K$ the conditions (a) - (d) hold and $f$ is a standard mapping. Since $f$ is scattering, there exists a bicompactification $b X \supset X$ and a scattering mapping

$$
F: b X \longrightarrow K
$$

such that the restriction of $F$ to $X$ is $f$ and the condition (e) holds. This result was proved in [23]. Since $X \subset b X$ we have

$$
\text { ind } X \leqq \text { ind } b X
$$

see (1) (introduction). Since every scattering mapping is obviously zero dimensional, we obtain by virtue of Lemma 5.1

$$
\begin{gathered}
D(K)=D(X) \geqq D(b X) \\
\omega_{0}+\text { ind } X \geqq \text { ind } K \geqq \text { ind } b X
\end{gathered}
$$

(4) ind $X=$ ind $K \geqq$ ind $b X$ if $X$ is strongly metrizable .

Thus, conditions (b)-(e) hold. Moreover we obviously can consider

$$
b X=\bar{X}
$$


where closures are taken in $b X$. Let us prove the inequality Ind $b X \leqq$ Ind $X$.

Since $f: X \rightarrow K$ is a standard mapping there is a standard representation

$$
X=P(X) \cup \bigcup_{i=1}^{\infty} C_{i}
$$

such that conditions (a), (b), (c) of Definition 3.2 hold. Let us prove the equality:

$$
b X=P(X) \cup \bigcup_{i=1}^{\infty} \bar{C}_{i} .
$$

Let $x \in b X \backslash P(X)$ be any point. Then there are open in $b X$ sets $V \supset$ $P(X)$ and $W \ni x$ such that

$$
\bar{V} \cap \bar{W}=\varnothing
$$

We put

$$
U=V \cap X \text {. }
$$

Then $U$ is a neighborhood of $P(X)$ in $X$. Since $P(X)$ is compact for some $\varepsilon>0$, we have:

$$
O_{\varepsilon}(P(X))=\{x: \delta(x, P(X))<\varepsilon\} \subset U .
$$

Since (7) is a standard representation of $X$, by virute of (c) (Definition 3.1) we have:

$$
C_{i} \subset U \text { for } 1 / i<\varepsilon .
$$

Consequently, by virtue of (9),

$$
\bar{W} \cap \overline{\left(P(X) \cup \bigcup_{j=k}^{\infty} \bar{C}_{j}\right)}=\varnothing \text { for any } k \text { such that } 1 / k<\varepsilon .
$$

This proves the condition (8) and shows that

$$
\text { the system }\left\{\bar{C}_{i}\right\} \text { is locally finite on } b X \backslash P(X) \text {. }
$$

Moreover from condition (b) of Defininition 3.2 it follows that

$$
\left.\overline{F\left(\bar{C}_{i}\right.}\right) \cap F(P(X))=\overline{F\left(\bar{C}_{i}\right)} \cap \overline{F\left(\bar{C}_{j}\right)}=\varnothing \text { for }|i-j|>1
$$

and consequently

$$
\bar{C}_{i} \cap \bar{C}_{j}=\varnothing=\bar{C}_{i} \cap P(X) \text { for }|i-j|>1 .
$$

From conditions (8), (10), (12) it follows that (see Definition 2.3), 
(13) the system $\left\{\bar{C}_{i}\right\}$ is simple with respect to $X \backslash P(X)$. Since $f$ is a standard mapping, we have:

$$
\operatorname{dim} \overline{f\left(C_{i}\right)}=\operatorname{dim} \overline{F\left(\bar{C}_{i}\right)<\infty,}
$$

and

(15) the restriction of $F=f$ to $P(X)$ is a homeomorphism.

From conditions (13), (11), (14), (15) it follows that conditions of Lemma 2.4 are fulfilled for $B=b X, B_{i}=\bar{C}_{i}$ and $P=P(X)$. Consequently by Lemma 2.4

$$
\text { Ind } b X \leqq \text { Ind } K=\text { Ind } X \text {. }
$$

If Ind $b X=X$ then our theorem is proved. Otherwise we can put

$$
b_{0} X=b X
$$

and consider the disjoint sum $b X=b_{0} X \cup K$ which obviously satisfies conditions (a)-(e).

7. Separable spaces which have no compactifications with the same dimension ind. By Theorem 6.1 (or by Corollary 1.1) every separable space $X$ with dimension Ind $X$ has a compactification $c X$ such that

$$
\text { ind } X=\text { ind } c X \text {. }
$$

In this section we construct examples of separable spaces which have no compactification with the same dimension ind. Similar examples for dimension $D$ we shall give in the next section.

Theorem 7.1. For any limit ordinal number $\alpha, \omega_{0} \leqq \alpha<\omega_{1}$, there exists such complete ${ }^{12}$ weakly-countable dimensional $l^{13}$ separable space $X_{\alpha}$ with dimension ind $X_{\alpha}=\alpha$ such that for any compactification $Y \supset X_{\alpha}$ we have

$$
\text { ind } Y>\alpha=\text { ind } X_{\alpha} \text {. }
$$

We note that by Hurewicz's theorem [5] every finite dimensional space $X$ has a compactification $c X \supset X$ such that ind $c X=$ ind $X$. It also follows from Corollary 1.1 because for finite dimensional space $X$ Ind $X=$ ind $X$.

12 We consider a space $X$ to be complete if it is an absolute $G_{\delta \text {-set }}$ or equivalently if we can introduce on $X$ a complete topology preserving metric.

13 A space $X$ is weakly countable dimensional if $X$ is a union of countable number of finite dimensional closed subsets. 
Definition 7.1. Let $\alpha$ be an ordinal number. We put

$$
\varphi(\alpha)=\alpha \text { for } \alpha \leqq \omega_{0} .
$$

If $\alpha>\omega_{0}$, then $\alpha=\omega_{0}+\beta$ for some $\beta>0$ and we put

$$
\varphi(\alpha)=\omega_{0}+\omega_{0} \times \beta .
$$

We will use the following results, see [13], §1, Luxemburg.

For any $\alpha<\omega_{1}$ there exists a weakly countable dimensional compactum $Y_{\alpha}$ such that:

$$
\text { ind } Y_{\alpha}=\alpha \operatorname{Ind} Y_{\alpha}=\varphi(\alpha) \text {. }
$$

(2) For any compactum $Y$ having a dimension ind $Y$ we have

$$
\text { ind } Y \leqq \operatorname{Ind} Y \leqq \varphi(\text { ind } Y) \text {. }
$$

From Definition 7.1 it follows that (see also [13, Lemma 1.1]).

$$
\begin{gathered}
\text { If } \alpha<\beta \text { then } \varphi(\alpha)<\varphi(\beta) . \\
\text { If } \alpha=\sup \left\{\beta_{s}\right\} \text { then } \varphi(\alpha)=\sup \left\{\varphi\left(\beta_{s}\right)\right\} .
\end{gathered}
$$

Construction of the space $X$. Since $\alpha$ is a limit number there exists such a sequence of ordinal numbers $\left\{\gamma_{i}\right\}$ so that

$$
\left\{\begin{array}{l}
\gamma_{i}<\gamma_{i+1}<\alpha, \quad \gamma_{i}=\beta_{i}+1 \\
\alpha=\sup \left\{\gamma_{i}: i=1,2, \cdots\right\}=\sup \left\{\beta_{i}: i=1,2, \cdots\right\}
\end{array}\right.
$$

By virtue of (1) there exists a weakly countable dimensional compacta $K_{i}$ such that

$$
\text { ind } K_{i}=\gamma_{i} \text {, Ind } K_{i}=\varphi\left(\gamma_{i}\right) \text {. }
$$

Since by virtue of (3), (5), $\varphi\left(\beta_{i}\right)<\varphi\left(\gamma_{i}\right)$ we can take in every compactum $K_{i}$ a pair of closed sets $F_{i}, G_{i}\left(F_{i} \cap G_{i}=\varnothing\right)$ such that

Any partition $C$ between $F_{i}$ and $G_{i}$ has the dimension Ind $C \geqq$ $\varphi\left(\beta_{i}\right)$.

Let $K_{i}^{\prime}$ be a compactum which we obtain by identification of all points of the $F_{i}$ with some point $n_{i} \in F_{i}$ and by identification of all points of the set $G_{i}$ with some point $v_{i} \in G_{i}$. Let

$$
Z=\bigcup_{i=1}^{\infty} K_{i}^{\prime}, \quad K_{i}^{i} \cap K_{j}^{\prime}=\varnothing \quad \text { for } \quad i \neq j
$$

be a discrete sum of compacta $K_{i}^{\prime}$. We put 


$$
U=\bigcup_{n=1}^{\infty}\left\{u_{n}\right\}, \quad V=\bigcup_{n=1}^{\infty}\left\{v_{n}\right\}, \quad\left(v_{n}, u_{n} \in K_{n}^{\prime}\right) .
$$

Then $U$ and $V$ are disjoint closed subsets in $Z$. We take a countable number of copies $Z_{p}$ of a space $Z$. The set in $Z_{p}$ which corresponds to a set $A \subset Z$ we shall denote by $A_{p}$. In the set $\bigcup_{p=1}^{\infty} Z_{p}$ with topology of a discrete union of copies $Z_{p}$ we identify the point $v_{p}^{n}$ with the point $u_{p+1}^{n}$ for all $n$ and $p$. Then we obtain the space

$$
\tilde{X}_{\alpha}=\bigcup_{p=1}^{\infty} Z_{p}
$$

such that

$$
Z_{p} \cap Z_{p+1}=V_{p}=U_{p+1}
$$

We put

$$
X_{\alpha}=\tilde{X}_{\alpha} \cup\{\delta\}
$$

where in a point $\delta$ we define the topology by the open basis

$$
G_{k+1}=X_{\alpha} \mid \bigcup_{p=1}^{k} Z_{p}
$$

and in $\tilde{X}_{\alpha}$ the topology is preserved.

Proof. From condition (7) it follows that

(10) any partition $C$ between $\left\{u_{i}\right\}$ and $\left\{v_{i}\right\}$ has the dimension Ind $C \geqq$ $\varphi\left(\beta_{i}\right)$.

Consequently Ind $K_{i}^{\prime}>\varphi\left(\beta_{i}\right)$ and by virtue of (2)

$$
\text { ind } K_{i}^{\prime} \geqq \beta_{i}+1=\gamma_{i} \text {. }
$$

Besides that

$$
\text { ind } K_{i}^{\prime} \leqq \gamma_{i}+1<\alpha \text {. }
$$

Indeed, from (6) it follows that for any point $x \in K_{i}^{\prime}\left(\left\{v_{i}\right\} \cup\left\{u_{i}\right\}\right)$

$$
\operatorname{ind}_{x} K_{i}^{\prime} \leqq \gamma_{i} \text {. }
$$

Further if $V$ is an arbitrary neighborhood of a point $v_{i}$ in $K_{i}^{\prime}$ and $\bar{V} \not u_{i}$ then

$$
F r V \subset K_{i}^{\prime} \backslash\left(\left\{v_{i}\right\} \cup\left\{u_{i}\right\}\right)
$$

and consequently ind $F r V \leqq \gamma_{i}$. Similarly one can prove that ind $_{v_{i}} K_{i}^{\prime} \leqq \gamma_{i}$. Hence (12) holds. From conditions (5), (8), (9), (11), (12) it follows that 


$$
\text { ind } \widetilde{X}_{\alpha}=\alpha \text {. }
$$

Since obviously $\operatorname{Fr} G_{k+1}=V_{k}=U_{k+1}$ and the set $V_{k}$ is countable, we have $\operatorname{ind}_{\bar{o}} X_{\alpha} \leqq 1$. Therefore

$$
\text { ind } X_{\alpha}=\alpha \text {. }
$$

Since compacta $K_{i}$ are weakly countable dimensional, compacta $K_{i}^{\prime}$ are also weakly countable dimensional. Consequently the space $X_{\alpha}$ is also weakly countable dimensional. Since $X_{\alpha}$ is a union of locally compact separable spaces $\widetilde{X}_{\alpha}$ and a point $\delta, X_{\alpha}$ is complete and separable. Let us prove that $X_{\alpha}$ is not contained in any compactum $Y$ with

$$
\text { ind } Y=\alpha \text {. }
$$

We suppose on the contrary. Then $Y \supset X_{\alpha}$ and (14) holds. By virtue of (2)

$$
\text { Ind } Y \leqq \varphi(\alpha) \text {. }
$$

We consider a neighborhood $O_{\hat{o}}$ in $Y$ of the point $\delta$ such that

$$
O_{\delta} \cap U_{1}=\varnothing \text {. }
$$

Since Ind $Y \leqq \varphi(\alpha)$, it follows that there exist neighborhoods $W_{i}, i=$ $1,2, \cdots$, of the point $\delta$ such that for all $i$

$$
\bar{W}_{i} \subset O_{\grave{o}}, \quad \bar{W}_{i} \subset W_{i+1}, \quad \text { ind } \operatorname{Fr} \bar{W}_{i}<\varphi(\alpha) .
$$

Since sets $G_{k}, k=2,3, \cdots$, constitute a basis in $X_{\alpha}$ at the point $\delta$, there is some integer $m$ such that $\bar{G}_{m} \subset W_{1}$. Thus for $k \geqq m$ the sets $U_{m}$ are contained in $W_{1}$. We shall show that $U_{m-1} \backslash \bar{W}_{2}$ contains only finitely many points. Indeed, if $u_{m-1}^{k_{1}}, u_{m-1}^{k_{2}}, \cdots$, is an infinite sequence of points which does not lie in $\bar{W}_{2}$, then since $u_{m}^{k_{1}}, u_{m}^{k_{2}}, \cdots \subset$ $U_{m} \subset \bar{W}_{m} \subset W_{2}$, we have that the set $\operatorname{Fr} \bar{W}_{2}$ separates points $u_{m-1}^{k_{i}}$ and $v_{m-1}^{k_{i}}$ and by virtue of (10)

$$
\text { Ind } \operatorname{Fr} \bar{W}_{2} \geqq \varphi\left(\beta_{k_{i}}\right) \text { for any } i \text {. }
$$

By virtue of (5) $\sup \left\{\beta_{k_{i}}: i=1,2, \cdots\right\}=\alpha$ and consequently by virtue of (4) $\sup \left\{\varphi\left(\beta_{k_{2}}\right): i=1,2, \cdots\right\}=\varphi(\alpha)$. Therefore from (17) it follows that

$$
\text { Ind } F r \bar{W}_{2} \geqq \varphi(\alpha)
$$

which contradicts the condition (16). Therefore, the set $U_{m-1} \backslash \bar{W}_{2}$ is finite. Analogously to the case $\ell=m-1$ we can show by induction that for $\iota=1,2, \cdots, m-1$ the set $U_{\ell} \backslash \bar{W}_{m+1-\iota}$ consists of only finitely many points. From this taking $\ell=1$ we get that the set $U_{1} \backslash \bar{W}_{m}$ is 
finite, but this contradicts conditions (15), (16). Thus, assumption of the existence of a compact space $Y \supset X_{\alpha}$ with ind $Y=\alpha=\operatorname{ind} X_{\alpha}$ leads us to a contradiction.

8. On compactifications of spaces with $D$-dimension. In [4], Henderson, it was proved that every separable weakly infinite dimensional space has a compactification of the same dimension $D$. This result also follows from Corollary 1.1. In that paper it was the conjecture which we prove in the following theorem:

THEOREM 8.1. Every separable space $X$ has a compactification $c X \supset X$ such that

$$
D(c X) \leqq D(X)+1^{14}
$$

This result appeared also in [7] earlier than ours. In [4] Henderson gave an example of separable space $X\left(D(X)=\omega_{0}\right)$ which is not contained in any compactum with the same D-dimension. In the following theorem this result will be generalized for all $\alpha<\omega_{1}$, $\alpha \geqq \omega_{0}$.

THEOREM 8.2. For any $\alpha, \omega_{0} \leqq \alpha<\omega_{1}$ there exists a separable space $X_{\alpha}$ such that $D\left(X_{\alpha}\right)=\alpha$ and $X_{\alpha}$ is not contained in any compactum with the same $D$-dimension.

To prove Theorem 8.1 we need some preliminary lemmas.

LEMMA 8.1. Let

$$
X=\cup\left\{A_{r}: \gamma \leqq J(\beta)\right\}
$$

be a $\beta$-D-representation of a separable space $X$. Then $X$ is homeomorphic to a subset of a separable space $Z$ such that for some $\beta-D$ representation of $Z$

$$
Z=\cup\left\{B_{r}: \gamma \leqq J(\beta)\right\}
$$

the set $B_{J(\beta)}$ is compact.

Proof. By virtue of Lemmas 1.4 and 3.3 there exists a homeomorphism $f: X \rightarrow I^{\omega}$ such that:

$$
\begin{aligned}
& \operatorname{dim} \overline{f\left(A_{J(\beta)}\right)} \leqq \operatorname{dim} A_{J(\beta)} \\
& \overline{f\left(A_{J(\beta)}\right)} \cap f\left(X \backslash A_{J(\beta)}\right)=\varnothing .
\end{aligned}
$$

${ }_{14}$ We will prove that it is possible to take such compactification $c X$ that $c X \in A R$ and inequality (1) holds. 
We put $Z=\overline{f\left(A_{J(\beta)}\right)} \cup f(X), B_{\gamma}=\overline{f\left(A_{r}\right)} \cap Z,(\gamma \leqq J(\beta))$. Then clearly $B_{J(\beta)}=f\left(A_{J(\beta)}\right)$ and the equality (3) is a needed $\beta$-D-representation of $Z$.

Lemma 8.2. Let (2) be a $\beta$-D-represntation of a space $X, \alpha=$ $J(\beta) \geqq \omega_{0}$, then for any sequence of ordinal numbers $\left\{\gamma_{i}\right\}$ such that

$$
\gamma_{i+1}>\gamma_{i}, \quad \sup \left\{\gamma_{i}: i=1,2, \cdots\right\}=\alpha
$$

there exists a simple with respect to $U=X \backslash A_{J(\beta)}$ system of sets $\mathscr{F}=\left\{F_{i}\right\}$ such that

$$
D\left(F_{i}\right) \leqq \gamma_{i}
$$

Proof. Let $\delta<\alpha$ then we put

$$
U=X \backslash\left\{A_{T}: \delta \leqq \gamma \leqq J(\beta)\right\}
$$

Then by Lemma 8.3 in [13], Luxemburg, $D\left(U_{\delta}\right)<\alpha$. From Definition 0.3 it follows that the sets $U_{\delta}$ are open and since the system $\left(U_{\gamma_{i}}: i=1,2, \cdots\right\}$ is countable, our lemma follows.

LEMMA 8.3. Let $P_{i}, i=1,2,3$, be clssed subsets of a space $P=$ $P_{1} \cup P_{2} \cup P_{3}$ and $P_{1} \cap P_{3}=\varnothing$. Let $f: P \rightarrow K$ be a homeomorphism. Then there exists a homeomorphism $g: P \rightarrow K \times I$, where $I=[0,1]$, such that $\overline{g\left(P_{1}\right)} \cap \overline{g\left(P_{3}\right)}=\varnothing$.

Proof. Let $\varphi: P \rightarrow[0,1]$ be a continuous function such that $\varphi\left(P_{1}\right)=1, \varphi\left(P_{3}\right)=0$. Then we put

$$
g(x)=\{f(x), \varphi(x)\}, \quad x \in P .
$$

Then, clearly, $g: P \rightarrow K \times I$ is a suitable mapping.

Definition 8.1. Let spaces $P, P_{i} i=1,2,3$, satisfy the condition of Lemma 8.3 and $h: P_{1} \cup P_{2} \rightarrow K$ is a homeomorphism in a compactum $K$ such that

$$
\overline{h\left(P_{1}\right)} \cap \overline{h\left(P_{2} \cap P_{3}\right)}=\varnothing .
$$

We put $T=\overline{h\left(P_{1}\right)} \cup h\left(P_{2}\right) \subset K$ and in disjoint sum $T \cup P_{3}$ we identify every point $h(x) \in T$ with a point $x$ for $x \in P_{2} \cap P_{3} \subset P_{3}$. Then we get a factor space, which we denote by $\mu\left(h, K, P_{1}, P_{2}, P_{3}\right)$.

Lemma 8.4. There exists an imbedding $\pi: P \rightarrow \mu\left(h, K, P_{1}, P_{2}, P_{3}\right)=$ $\mu$. Moreover, the space $\mu$ is separable and metrizable if $P$ is separable and metrizable, $\pi(P)$ is everywhere dense in $\mu$, 


$$
\left.\mu=\overline{\pi\left(P_{1}\right.}\right) \cup \pi\left(P_{2}\right) \cup \pi\left(P_{3}\right)
$$

the set $\pi\left(P_{3}\right)$ is closed in $\mu, \pi\left(P_{1}\right)$ is compact, $\overline{\pi\left(P_{1}\right)} \cup \pi\left(P_{2}\right)$ is closed in $\mu$ and can be imbedded in $K$ and $\overline{\pi\left(P_{1}\right)} \cup \pi\left(P_{3}\right)=\varnothing$. The lemma is evident.

We will consider the following conjecture:

$\mathscr{C}(\alpha)$. For any separable space $X$ with $D(X)<\alpha$ there exists a compactification $K \supset X$ with $D(K)<\alpha$.

DeFinition 8.2. Let $X=\cup\left\{X_{i}: i \in I\right\}$ be a union of spaces $X_{i}$ then $X$ is called an inductive limit $X=\operatorname{Lim}\left\{X_{i}\right\}$ if a set $U \subset X$ is open in $X \Leftrightarrow U \cap X_{i}$ is open in $X_{i}$.

LEMma 8.5. Let $\alpha$ be a limit ordinal number and conjecture $C(\alpha)$ is ture. Let the system $\left\{F_{i}\right\}(i=1,2, \cdots)$ in a separable space $X$ be simple with respect to $X$ and $D\left(F_{i}\right)<\alpha$. Then there exists $a$ locally compact separable space $G$, the system of compacta $\left\{G_{i}\right\}$ in $G$ and imbedding $f: X \rightarrow G$ such that:

(a) $\overline{f\left(F_{i}\right)}=G_{i}$.

(b) $G_{i}$ is a compactum.

(c) The system $G_{i}$ is simple with respect to $G$.

(d) $D\left(G_{i}\right)<\alpha, D(G) \leqq \alpha$.

Proof. We will define by induction spaces $Y_{i} \supset X, i=0,1,2, \cdots$ such that

$$
\begin{gathered}
Y_{i}=P_{0} \cup \cdots \cup P_{i} \cup F_{i+1} \cup F_{i+2} \cup \cdots \\
\bar{F}_{j}=P_{j} \text { and } P_{j} \text { is compact } j=0,1, \cdots, i .
\end{gathered}
$$

where $\bar{F}_{j}$ is a closure of $F_{j}$ in $Y_{i}$.

$$
\bar{Q}_{1}^{i} \cap \bar{Q}_{3}^{i}=\varnothing, \quad Q_{1}^{i}=\bar{Q}_{1}^{i}, \quad Q_{3}^{i}=\bar{Q}_{3}^{i}, \quad F_{i+2}=\bar{F}_{i+2}
$$

where $Q_{1}^{i}=\bigcup_{k=0}^{i} P_{k} \cup F_{i+1}, Q_{3}^{i}=\bigcup_{j=i+3}^{\infty} F_{j}, Q_{2}^{i}=F_{i+2}$.

$$
\begin{gathered}
P_{k} \cap P_{\ell}=P_{k} \cap F_{\ell}=\varnothing \text { if }|k-\ell|>1 . \\
D\left(Q_{1}^{i}\right)<\alpha .
\end{gathered}
$$

We put $P_{0}=F_{0}=\varnothing, Y_{0}=X$. Let a space $Y_{i}$ for $i=i_{0}$ be defined. We put $Q_{2}^{i}=F_{i+2}$. Then from (6) it follows that sets $Q_{1}^{i}, Q_{2}^{i}, Q_{3}^{i}$ are closed in $Y_{i}$. Since $D\left(F_{k}\right)<\alpha$, from condition (8) and the sum theorem for $D$-dimension (see [3], Henderson), it follows that $D\left(Q_{1}^{i} \cup Q<\right.$ $\alpha$. Consequently, by $C(\alpha)$ there exists an imbedding $f: Q_{1}^{i} \cup Q_{2}^{i} \rightarrow K$ in a compactum $K$ with $D(K)<\alpha$. From Lemma 3.3 it follows that 
there exists an imbedding $\psi: Q_{1}^{i} \cup Q_{2}^{i} \rightarrow K \times I$ such that $\overline{\psi\left(Q_{1}^{i}\right) \cap}$ $\overline{\psi\left(Q_{2}^{i} \cap Q_{3}^{i}\right)}=\varnothing$. We put $Y_{i+1}=\mu\left(\psi, K \times I, Q_{1}^{i}, Q_{2}^{i}, Q_{3}^{i}\right), P_{i+1}=\left[F_{i+1}\right]$, where $\left[F_{i+1}\right]$ is a closure of $F_{i+1}$ in $Y_{i+1}$. Conditions (4), (5), (6), (7), for $i_{0}+1$ follow from Lemma 8.4. Since by Lemma 8.4 $Q_{1}^{i_{0}+1}=Q_{1}^{i_{0}+1} U$ $Q_{2}^{i_{0}}$ can be embedded in $K \times I$, we have $D\left(Q_{1}^{i_{0}+1}\right)<D(K \times I)$. Since $\alpha$ is a limit number, property (8) follows from the inequality $D(K \times I) \leqq$ $D(K)+1$ (see [3], Henderson). Hence, space $Y_{i}$ are constructed. Let us put

$$
G=\lim \left\{Y_{i}\right\}, \quad G_{i}=\pi_{i}\left(P_{i}\right)
$$

where $\pi_{i}: Y_{i} \rightarrow G$ are inclusions. From conditions (6), (7) it follows that for every point $x \in G$ there exists $i$ and an open in $Y_{i}$ set $U, x \in U \subset P_{j} \cup P_{j+1}(j+1 \leqq i)$, such that $\pi_{i}(U)$ is a neighborhood of $x$ in $G$. Consequently, by virtue of (7) we obtain the condition (c). Conditions (a), (b) follow from (5). Since $\pi_{i}$ is a homeomorphism, inequality $D\left(G_{i}\right)<\alpha$ follows from inclusion $P_{j} \subset Q_{1}^{i}(j<i)$ and (8). Since collection of compacta $\left\{G_{i}\right\}$ is locally finite, the inequality $D(G) \leqq \alpha$ follows from sum theorem for $D$-dimension (see [3], Henderson). Hence property (d) and Lemma 8.5 are proved.

Notation. Let $K \subset Y, X \subset Y, K$ is compact and $f: K \rightarrow R$ is a mapping in a compact space $R$, such that if $x \in K \cap X$ then for any $y \in K, y \neq x$, we have $f(x) \neq f(y)$. We will consider points $x, y \in Y$ to be equivalent if either $x=y \in Y \backslash K$ or $f(x)=f(y)$ and $x, y \in K$. We get a factor space which we will denote by $F=F(K, X, Y, f)$ and a factor mapping $\pi: Y \rightarrow F$.

LEMMA 8.6. If a space $Y$ is metrizable and separable then $F$ is also metrizable and separable. Moreover, the restriction of $\pi$ to $X$ is a homeomorphism.

The lemma follows from well known theorems on factor mappings.

Definition 8.2. The equality

$$
Y=K \cup \bigcup_{i=1}^{\infty} F_{i}
$$

we will call canonic representation of a space $Y$ if

(a) the system $\left\{F_{i}\right\}$ is simple with respect to $Y \backslash K$.

(b) any sequence of points $\left\{x_{i}\right\}$ such that $x_{i} \in F_{i(k)}, i(k+1)>$ $i(k)$, has an accumulation point $y \in K$.

LEMmA 8.7. Let $X$ be a separable space and (2) be a $\beta$ - $D$ representation of $X\left(\beta \geqq \omega_{0}\right)$. Then there exists a separable space $Y \supset X$ such that $Y$ has a canonic representation (9) and 
(i) $K$ is compact.

(ii) $D\left(F_{i}\right)<\alpha=J(\beta)$.

(iii) $K \cap X=A_{J(\beta)}$.

Proof. By Lemma 8.2 there exists a simple with respect to $V=$ $X \backslash A_{J(\beta)}$ system $\left\{F_{i}^{\prime}\right\}$ in a space $X$ such that

$$
D\left(F_{i}^{\prime}\right)<\alpha .
$$

Since $\left\{F_{i}^{\prime}\right\}$ is a simple system, sets $F_{i}^{\prime}$ and $P_{i}=A_{J(\beta)} \cup \cup\left\{F_{k}^{\prime}:|k-i|>1\right\}$ are closed and disjoint. Since the set of all homeomorphisms $\varphi: X \rightarrow$ $I^{\omega}$ contains an everywhere dense $G_{\delta}$-set in $C\left(X, I^{\omega}\right)$, we get by Lemma 1.2 and (19) 11 a homeomorphism $f: X \rightarrow I^{\omega}$ such that

$$
\overline{f\left(P_{i}\right)} \cap \overline{f\left(F_{i}^{1}\right)}=\varnothing \text { for any } i \text {. }
$$

We put

$$
\begin{gathered}
K_{i}=\overline{\bigcup_{j=1}^{\infty} f\left(F_{j}\right) \cup A_{J(\beta)}} \subset I^{\omega}, \quad K=\bigcap_{i=1}^{\infty} K_{i}, \quad F_{i}=f\left(F_{i}^{\prime}\right) \\
Y=K \cup \bigcup_{i=1}^{\infty} F_{i} .
\end{gathered}
$$

Since $f$ is a homeomorphism, $Y \supset f(X)$, we can consider that $X=$ $f(X)$. Then conditions (i)-(iii) follow from (10), (11), (12), (13). Let us prove that representation (13) is canonic. Property (a) of Definition 8.2 follows from (11). Let $\left\{x_{i}\right\}$ be such a sequence as in (b) of Definition 8.2. Then $x_{i} \subset F_{i(k)} \subset K_{i(k)}$. Since $K_{i+1} \subset K_{i}$ and $K_{i}$ are compact, there is an accumulation point $y \in \bigcap_{i=1}^{\infty} K_{i}=K$. The lemma is proved.

Notation. It is known (see [1], Bothe) that every separable $n$-dimensional space is contained in compact separable $(n+1)$-dimensional $A R$-space $R^{n+1}$. In particular $R^{n+1}$ contains a universal $n$ dimensional compact space $A_{n} \subset R^{n+1}$.

LEMMA 8.8. In Lemma 8.7 we can also require that $K=R^{n+1}$, $R^{n+1} \cap X=A_{J(\beta)} \subset A_{n}$, where $n=K(\beta)$.

Proof. By Lemma 8.1 we can consider the set $A_{J(\beta)}$ to be a compactum. Let $Y$ be a space from Lemma 8.7. Since $\operatorname{dim} A_{J(\beta)} \leqq$ $K(\beta)=n$ (see Definition $0.3(\mathrm{~d})$ ), there is a homeomorphism $g: A_{J(\beta)} \rightarrow$ $A_{n} \subset R_{n+1}$. Let $f: K \rightarrow R_{n+1}$ be an extension of $g$. We put $Y^{\prime}=$ $F(K, X, Y, f)$. Then by Lemma 8.6 we can consider that $X \subset Y^{\prime}$, and the equality

$$
Y^{\prime}=R^{n+1} \cup \bigcup_{i=1}^{\infty} F_{i}
$$


is clearly a canonic representation of $Y^{\prime}$. Condition

$$
R_{n+1} \cap X=A_{J(\beta)}
$$

is evident.

LEMMA 8.9. Let $X$ be a separable space, $D(X) \leqq \beta, \beta=\alpha+n$, $\alpha=J(\beta), n=K(\beta)$. Then there exists a compact space $K \supset X$ such that

(i) $D(K) \leqq \beta+1=\alpha+n+1$.

(ii) $\overline{K=} R^{n+1} \cup \bigcup_{i=1}^{\infty} H_{i}, H=\bigcup_{i=1}^{\infty} H_{i}, H \cap \overline{R^{n+1}}=\varnothing$.

(iii) $D\left(H_{i}\right)<\alpha, D(H) \leqq \alpha$. to $H$.

(iv) $H_{i}$ is compact and the system $\left\{H_{i}\right\}$ is simple with respect

(v) There exists a homeomorphism $i: \overline{R^{n+1}} \rightarrow R^{n+1}$ such that $i\left(X \cap \overline{R^{n+1}}\right) \subset A_{n} \subset R^{n+1}$.

Proof. We shall prove our theorem by induction on $\beta$. If $\beta<$ $\omega_{0}$ then we put $K=R^{n+1}, G=\varnothing$. Let $\beta \leqq \omega_{0}$. Since $D(X) \leqq \beta$, there exists a $\beta$-D-representation of $X$ and by Lemma 8.8 there exists a separable space $Y^{\prime} \supset X$ such that representation (14) is canonic and

$$
X \cap R^{n+1} \subset A_{n}, \quad D\left(F_{i}\right)<\alpha .
$$

Since the conjecture $C(\alpha)$ holds by inductive assumption, by Lemma 8.5 there exists a separable locally compact space $G$ such that $\bigcup_{i=1}^{\infty} G_{i}=G, G_{i}$ is compact, the system $\left\{G_{i}\right\}$ is simple with respect to G.

$$
D\left(G_{i}\right)<\alpha, \quad D(G) \leqq \alpha
$$

and there exists a homeomorphism $h: Y^{\prime} \backslash R^{n+1} \rightarrow G$ such that $h\left(F_{i}\right) \subset$ $G_{i}$. Let us put

$$
G^{\prime}=\omega \cup G
$$

where $\omega$ is a compactification point and $G^{\prime}$ is a compactum. Then obviously $D\left(G^{\prime}\right)=D(G)$. We put:

$$
K=R^{n+1} \times G^{\prime}, H_{i}=R^{n+1} \times G_{i}, H=R^{n+1} \times G, \overline{R^{n+1}}=R^{n+1} \times \omega .
$$

Then from (16), (17) it follows that

$$
D(K)=D\left(G^{\prime}\right) \oplus D\left(R^{n+1}\right)^{15}=D(G) \oplus(n+1) \leqq \alpha+n+1=\beta+1 .
$$

Since $\alpha$ is a limit number from (16), (17) it follows that

${ }^{15}$ Here we use the inequality $D(X \times Y) \leqq D(X) \oplus D(Y)$, where $\oplus$ is a natural sum of ordinal numbers, see [3], Henderson. 


$$
D\left(H_{i}\right) \leqq D\left(G_{i}\right) \oplus D\left(R^{n+1}\right)=D\left(G_{i}\right)+n+1<\alpha .
$$

Moreover the system $H_{i}$ is simple with respect to $H$ because the system $\left\{G_{i}\right\}$ simple with respect to $G$. Consequently, from (18) and the sum theorem for $D$-dimension (see [3]) it follows that $D(H) \leqq \alpha$. Thus conditions (i)-(iv) of our lemma are satisfied. We have only to prove the existence of homeomorphism $F: Y^{\prime} \rightarrow K$ such that

$$
F(X) \cap \overline{R^{n+1}} \subset A_{n} \times \omega .
$$

Then condition (v) will take place. Since $R^{n+1}$ is $A R$ space, there exists a retraction $r: Y^{\prime} \rightarrow R^{n+1}$. We define a mapping $q: Y^{\prime} \rightarrow G^{\prime}$ by the equalities

$$
q(y)=h(y) \text { for } y \in Y^{\prime} \backslash R^{n+1}, \quad q(y)=\omega \in G^{\prime} \text { for } y \in R^{n+1} .
$$

Then obviously $q$ is a homeomorphism on $Y^{\prime} \backslash R^{n+1}$. We define a mapping $F: Y^{\prime} \rightarrow K$ by the equalities:

$$
\pi_{1} \circ F(y)=q(y), \quad \pi_{2} \circ F(y)=r(y)
$$

where $\pi_{1}: K=R^{n+1} \times G^{\prime} \rightarrow G^{\prime}, \pi_{2}: K \rightarrow R^{n+1}$ are projections. The condition (19) follows from (15). Let us prove that $F$ is a homeomorphism. The mapping $F$ is obviously injective and continuous. Moreover, since $q$ is a homeomorphisms on $Y^{\prime} \backslash R^{n+1}$ and $r$ is a homeomorphism on $R^{n+1} \subset Y^{\prime}$, the mapping $F$ is a homeomorphism on $Y^{\prime} \backslash R^{n+1}$ and on $R^{n+1}$. Besides that

$$
F\left(Y^{\prime} \backslash R^{n+1}\right) \subset H, \quad F\left(R^{n+1}\right)=\overline{R^{n+1}}, \quad F\left(Y^{\prime} \backslash R^{n+1}\right) \cap F\left(R^{n+1}\right)=\varnothing .
$$

Therefore, for proving that $F$ is a homeomorphism, we have only to prove that for any sequence $\left\{F\left(x_{n}\right)\right\}$ such that

$$
F\left(x_{n}\right) \in H, \lim _{n \rightarrow \infty} F\left(x_{n}\right)=F(y) \in \overline{R^{n+1}}
$$

we have

$$
\lim _{n \rightarrow \infty} x_{n}=y .
$$

From condition (20) it follows that $\lim _{n \rightarrow \infty}\left(\pi_{1} \circ F\left(x_{n}\right)=q\left(x_{n}\right)=h\left(x_{n}\right)\right)=$ $\pi_{1} \circ F(y)=\omega$. Since $G_{i}$ and $G^{\prime}=\pi_{1}(K)$ are compact, there exist two subsequences of natural numbers $\{n(k)\},\{i(k)\}$ such that

$$
h\left(x_{n(k)}\right) \subset G_{i(k)}, \quad n(k+1)>n(k)+2, \quad i(k+1)>i(k)+2 .
$$

Since $h\left(F_{i}\right) \in G_{i}$ and systems $\left\{G_{i}\right\},\left\{F_{i}\right\}$ are simple [from condition (22)], it follows that

$$
x_{n(k)} \subset F_{i(k)-1} \cup F_{i(k)} \cup F_{i(k)+1} .
$$


Let $j(k)$ is such a number so that $x_{n(k)} \in F_{j(k)}$, then since $\left\{F_{i}\right\}$ is a simple system, from conditions (22), (23) it follows that $j(k+1)>$ $j(k)$. Since $(14)$ is a canonic representation, a sequence $\left\{x_{n(k)}\right\}$ has an accumulation point $y^{\prime} \in R^{n+1}$ and consequently $y^{\prime}=\lim \left\{z_{j}\right\}$ for some subsequence $\left\{z_{j}\right\}$ of the sequence $\left\{x_{n(k)}\right\}$. Moreover $F\left(y^{\prime}\right)=F(y)$ and since $F$ is injective, we have $y=y^{\prime}$. We have proved that for any sequence with condition (20) there exists a subsequence $\left\{z_{j}\right\}$ of a sequence $\left\{x_{n}\right\}$ such that $\lim z_{j}=y$. From this fact it follows (21). The lemma is proved.

Theorem 8.1 obviously follows from Lemma 8.9. Let us prove Theorem 8.2.

Definition 8.3. (See [20], Smirnov). For any ordinal number $\beta<\omega_{1}$ we shall define a compactum $K_{\beta}$. For $\beta<\omega_{0} K_{\beta}$ is a $\beta$ dimensional cube. If $\beta$ is a limit number we consider $K_{\beta}$ to be a one-point compactification (with point $p_{\beta}$ ) of a discrete union of compact $K_{r}: \gamma<\beta$. If $\beta=\alpha+n, \alpha=J(\beta), n=K(\beta)$, we put $K_{\beta}=$ $K_{\alpha} \times I^{n}$, where $I^{n}$ is an $n$-dimensional cube.

In what follows we will consider $\alpha$ to be a limit number $<\omega_{1}$ and $n=0,1,2, \cdots$. For any $K_{\beta}$

$$
\text { Ind } K_{\beta}=\beta \text {. }
$$

(See [20], Smirnov.) Since for any compactum $Z$

$$
D(Z) \geqq \operatorname{Ind} Z,
$$

see [4], Henderson (Theorem 2), $D\left(K_{\beta}\right) \geqq$ Ind $K_{\beta}=\beta$. By transfinite induction it is easy to prove that $D\left(K_{\beta}\right) \leqq \beta$. Therefore

$$
D\left(K_{\beta}\right)=\beta \text {. }
$$

By definition we have

$$
K_{\alpha+n+1}=\left\{p_{\alpha}\right\} \times I^{n+1} \cup \cup\left\{K_{r} \times I^{n+1}: \gamma<\alpha\right\} .
$$

Let $S^{n}$ be a sphere which is a boundary of the cube $I^{n+1}$. We put:

$$
X_{\alpha+n}=\left(\left\{p_{\alpha}\right\} \times S^{n}\right) \cup \cup\left\{K_{\gamma} \times I^{n+1}: \gamma<\alpha\right\} \subset K_{\alpha+n+1} .
$$

Then $X_{\alpha+n}=Y \cup\left(\left\{p_{\alpha}\right\} \times S^{n}\right)$, where $Y$ is a discrete union of compacta $K_{\gamma} \times I^{n+1}: \gamma<\alpha$. From (26) it follows that

$$
D(Y) \leqq \sup \left\{D\left(K_{\gamma} \times I^{n+1}\right): \gamma<\alpha\right\}=\alpha .
$$

Since $Y$ is open in $X_{\alpha+n}$ and $\left\{p_{\alpha}\right\} \times S^{n}$ is closed we have the inequality (see [3], Henderson, Theorem 4) 


$$
D\left(X_{\alpha+n}\right) \leqq D(Y)+D\left(p_{\alpha} \times S^{n}\right)=\alpha+n .
$$

Let $A$ be an $n$-dimensional face of the cube $I^{n+1}$, then $K_{\alpha} \times A \subset$ $X_{\alpha+n} \subset K_{\alpha+n+1}$ and $K_{\alpha} \times A$ is homeomorphic to $K_{\alpha+n}$. Consequently

$$
D\left(X_{\alpha+n}\right) \geqq D\left(K_{\alpha} \times A\right)=D\left(K_{\alpha+n}\right)=\alpha+n .
$$

Thus $D\left(X_{\alpha+n}\right)=\alpha+n$. Let $\left\{A_{i}, B_{i}\right\} i=1, \cdots, n+1$ be a system of all pairs of opposite faces in cube $I^{n+1}$, then clearly $\left\{K_{\alpha} \times A_{i}, K_{\alpha} \times B_{i}\right\}$ is a system of compact disjoint pairs in $X_{\alpha+n}$. Let $C \supset X_{\alpha+n}$ by any compact space, then we have to prove that

$$
D(C) \geqq \alpha+n+1=\beta+1 .
$$

We put $F_{i}=K_{\alpha} \times A_{i}, G_{i}=K_{\alpha} \times B_{i}$. Let $D_{i}$ be a partition between $F_{i}$ and $G_{i}$ in $C$.

Since clearly

$$
F_{i} \cap K_{r} \times I^{n+1}=K_{r} \times A_{i}, \quad G_{i} \cap K_{r} \times I^{n+1}=K_{\gamma} \times B_{i}(\gamma<\alpha)
$$

the set $C_{i}^{\gamma}=D_{i} \cap K_{\gamma} \times I^{n+1}$ is a partition between $K_{\gamma} \times A_{i}$ and $K_{\gamma} \times$ $B_{i}$ in $K_{r} \times I^{n+1}=K_{r+n+1}$ for all $\gamma<\alpha$. Consequently, by virtue of [13], Luxemburg, Lemma 4.8 we have for all $\gamma<\alpha$

$$
\text { Ind }\left[\bigcap_{i=1}^{n+1} C_{i}=\left[\bigcap_{i=1}^{n+1} D_{i} \cap K_{\gamma} \times I^{n+1}\right]\right] \geqq \gamma .
$$

Therefore Ind $\left(\bigcap_{i=1}^{n+1} D_{i}\right) \geqq \sup \{\gamma: \gamma<\alpha\}=\alpha$ and by virtue of [13], Luxemburg, Lemma 4.7

$$
\text { Ind } C \geqq \alpha+n+1=\beta+1 \text {. }
$$

Inequality (29) now follows from (25), (30). The theorem is proved.

9. On completion of metric spaces. It is known, see [15], Nagata, that every finite dimensional subspace $X \subset Y$ is contained in $G_{i}$-set $G \subset Y$ with the same dimension Ind $G=\operatorname{Ind} X$. Here we extend this result to infinite dimensional case.

TheOREM 9.1. For any subspace $X$ of a space $Y$, such that the dimension Ind $X$ exists, there is a $G_{i}$-set $G$, such that Ind $G=$ Ind $X$, $X \subset G \subset Y, P(X)=P(G)$.

We note that by Lemma 2.8 we have also $D(X)=D(G)$.

Corollary 9.1. For any space $X$ with dimension Ind $X$ there exists a completion $G=X^{16}$ such that Ind $X=$ Ind $G$, weight $X=$

18 We call a space $G \supset X$ a completion of a space $X$ if $G$ is an absolute $G_{\delta}$-set. It is equivelant to the fact that we can introduce in $G$ a complete metric (see [8]). 
weight $G$.

Proof. Let $Y \supset X$ be any complete space and $G_{0}$ be a $G_{\delta}$-set in $Y$ such that $X \subset G_{0} \subset Y$, Ind $G_{0}=$ Ind $X$. Such $G_{0}$ exists by Theorem 9.1. We put $G=G_{0} \cap \bar{X}$. Then $G$ is $G_{\delta}$ in $Y$, and consequently is an absolute $G_{\dot{\delta}}$-set. Also, Ind $G \leqq \operatorname{Ind} G_{0}=\operatorname{Ind} X$. Inequality Ind $G \geqq$ Ind $X$ follows from Corollary 2.3 and (SM1) $\S 2$.

For proving Theorem 9.1 we need some preliminary results.

(S2) [19], Sklyarenko. If in a space $X$ there exists a convergent system (see Definition 2.2) of weakly infinite dimensional open sets with weakly infinite dimensional limit then $X$ is weakly infinite dimensional.

LEMMA 9.1. Let $K(K \subset X)$ be a limit of a system of open sets $\left\{\Gamma_{n}\right\}$ in a space $X$, then if $K \subset Y \subset X$ the system $\left\{\Gamma_{n} \cap Y\right\}$ is convergent in $Y$ and $K$ is a limit of this system in $Y$.

The lemma is evident.

LeMma 9.2. Let a space $X$ have the dimension Ind $X$ and $Y \subset X$ is a subspace such that $Y \supset P(X)$. Then the dimension Ind $Y$ exists and Ind $Y \leqq$ Ind $X$ and $Y$ is weakly infinite dimensional.

Proof. Let $U_{n}$ be the set defined by equality (1) $\$ 2$. Since Ind $X$ exists, the space $X$ is weakly infinite dimensional by (SM1) $\S 2$, consequently by Theorem (S1) $\S 2$ the set $X \backslash \bigcup_{n=1}^{\infty} U_{n}=P(X)$ is a limit of the system $\left\{U_{n}\right\}$. By Lemma $9.1 P(X)$ is a limit of the system $\left\{U_{n} \cap Y\right\}$. Since $\operatorname{dim} U_{n} \cap Y \leqq \operatorname{dim} U_{n} \leqq n$ sets $U_{n} \cap Y$ are weakly infinite dimensional. Moreover $P(X)$ is closed in $X$ and, consequently, is also weakly infinite dimensional. Therefore, by virtue of (S2) $Y$ is weakly infinite dimensional. Our lemma now follows from Corollary 2.3.

Proof of Theorem 9.1. We shall prove this theorem by induction on Ind $X$. If Ind $X$ is a finite number then this theorem is known (see [15]), and in this case $P(X)=\varnothing$. Let Ind $X \geqq \omega_{0}$. Then $P(X)$ is a nonempty compactum (Corollary 2.1). We put

$$
V_{n}=Y \backslash \overline{O_{1 / n} P(X)} \text {. }
$$

Then by Theorem SM1 $\S 2$ and by Corollary 2.1 the set $V_{n} \cap X$ is finite dimensional. Then by inductive assumption there exists $G_{j}$-sets $G_{n}$ such that 


$$
V_{n} \supset G_{n} \supset V_{n} \cap X, \text { Ind } G_{n}<\omega_{0} .
$$

We put

$$
F=\bigcup_{n=1}^{\infty}\left(V_{n} \backslash G_{n}\right), \quad D_{n}=(Y \backslash F) \cap V_{n} .
$$

Then $F$ is $F_{\sigma}$ set in $Y, D_{n}$ is open in $Y \backslash F$ and

$$
X \subset Y \backslash F, \quad D_{n} \subset G_{n} .
$$

Consequently, by virtue of (2), $D_{n}$ is finite dimensional. By virtue of (3) we have

$$
\phi=P(X) \cup \bigcup_{n=1}^{\infty} D_{n}=Y \backslash F .
$$

Since $P(X)$ is compact, by virtue of (1), (3) the system $\left\{D_{n}\right\}$ is convergent in $\phi$ and $P(X)$ is a limit of this system. Since $P(X)$ is closed in $X$, it is weakly infinite dimensional. Since $D_{n}$ are finite dimensional and consequently weakly infinite dimensional, $\phi$ is also weakly infinite dimensional by

THEOREM S2. Since $F$ is $F_{\sigma}$-set, $\phi=Y \backslash F$ is a $G_{\dot{o}}$-set in $Y$. Moreover, since $X \subset \phi$ and $D_{n}$ are fininite dimensional, we have

$$
P(\phi)=P(X) \text {. }
$$

Let Ind $X=\alpha \geqq \omega_{0} \gamma$ then there exists a countable system $\left\{U_{k}\right\}$ of open in $Y$ sets such that a system $\left\{U_{k}\right\}$ forms a large base in $P(X)$ and

$$
\text { Ind } \operatorname{Fr} U_{k} \cap X \leqq \beta_{k}<\alpha \text {. }
$$

By inductive assumption for any $k$ there exists such $G_{o}$-set $W_{k}$ in $Y$ so that

(8) $\quad W_{k} \supset F r U_{k} \cap X, \quad$ Ind $W_{k} \leqq \beta_{k}, \quad P\left(W_{k}\right)=P\left(F r U_{k} \cap X\right)$.

We put $H_{K}=F r U_{K} \backslash W_{K}$, then $H_{K}$ is $F_{\sigma}$-set in $Y$ and $H_{K} \cap X=\varnothing$. Consequently the set $G=\phi \backslash \bigcup_{k=1}^{\infty} H_{K}$ is a $G_{\tilde{o}}$-set and

$$
X \subset G, \quad \operatorname{Fr} U_{k} \cap X \subset \operatorname{Fr} U_{k} \cap G \leqq W_{k} .
$$

From conditions (8), (9) it follows that $P\left(F r U_{k} \cap X\right)=P\left(F r \cdot U_{k} \cap G\right)=$ $P\left(W_{k}\right)$ and by Lemma 9.2 we have

$$
\text { Ind Fr } U_{k} \cap G \leqq \operatorname{Ind} W_{k} \leqq \beta_{k}<\alpha \text {. }
$$

Since $X \subset G \subset \phi$, by virtue of (6) we have

$$
P(G)=P(X)=P(\phi) \text {. }
$$


Consequently by Lemma $9.2 \mathrm{G}$ is weakly infinite dimensional, because $\phi$ is weakly infinite dimensional.

Since $\left\{V_{k}\right\}$ is a large base in $P(G)$, for any closed subsets $F_{1}, F_{2}$ in $P(G)$ there exists a set $U_{k}$ such that $\operatorname{Fr} U_{k} \cap G$ is a partition in $G$ between $F_{1}$ and $F_{2}$. Therefore from condition (10) and Lemma 2.5 it follows that

$$
\text { Ind } G \leqq \alpha .
$$

The inequality Ind $G \geqq$ Ind $X=\alpha$ follows from Corollary 2.3.

THEOREM 9.2. Let $X$ be a separable subspace of a space $Y$, and the dimension ind $X$ exists. Then there exists a $G_{i}$-set $G \subset Y$ such that ind $G=$ ind $X, X \subset G$.

Problem. Is this theorem true for a nonseparable space? The answer is still unknown even for finite dimensional spaces.

COROLlaRy 9.2. For any separable space $X$ with dimension ind $X$ there exists a separable completion $G \supset X$ with the same dimension ind $G=$ ind $X$.

The proof is similar to the one of Corollary 9.1.

Proof of Theorem 9.2. We shall prove this theorem by induction on ind $X$. For ind $X=-1$ the theorem is evident. Let ind $X=$ $\alpha>-1$. Since $X$ is separable for any $n=1,2, \cdots$, there exists a countable system of open in $Y$ sets $\left\{V_{n i}\right\}$ such that

$$
\text { ind }\left(\operatorname{Fr} V_{n i}\right) \cap X \leqq \beta_{n i}<\alpha
$$

and

$$
X \subset H_{n}=\bigcup_{i=1}^{\infty} V_{n i}, \quad \operatorname{diam} V_{n i}<\frac{1}{n}
$$

By inductive assumption for every pair $(i, n)$ there exists a set $G_{i n}$ of a type $G_{\delta}$ in $Y$ such that

$$
F r V_{n i} \cap X \subset G_{n k}, \quad \text { ind } G_{n i}=\operatorname{ind}\left(F r V_{n i} \cap X\right)=\beta_{n i}<\alpha .
$$

We put

$$
A_{n}=H_{n} / \bigcup_{i=1}^{\infty}\left(F r V_{n i} \mid G_{n i}\right), \quad G=\bigcap_{n=\mathfrak{\tau}}^{\infty} A_{n} .
$$

Then $A_{n} \supset X$ and $A_{n}$ is a $G_{\delta}$-set in $Y$. Consequently $G \supset X$ and $G$ is also a $G_{\delta}$-set in $Y$. Let us prove the inequality 


$$
\text { ind } G \leqq \alpha .
$$

Let $x \in G, \varepsilon>0$ and $1 / n<\varepsilon$ for some $n$. Then by virtue of (11) there exists an open set $V_{n i}$ such that $x \in V_{n i}$. From (12) and (13) it follows that

$$
\operatorname{Fr} V_{n i} \cap G \subset \operatorname{Fr} V_{n i} \cup A_{n} \subset G_{n i}
$$

and consequently ind $\left(F r V_{n i} \cap G\right) \leqq$ ind $G_{n i}<\alpha$. Hence inequality (14) is true. Since $X \subset G$ we also have ind $G \geqq$ ind $X$.

THEOREM 9.3. Let $X \subset Y$ then there exists a $G_{j}$-set $G$ in $Y$ such that $D(X)=D(G), X \subset G$.

CoROllary 9.3. For any space $X$ there exists a completion $G \supset X$ such that $D(G)=D(X)$, weight $G=$ weight $X$.

The proof is similar to the one of Corollary 9.1.

Lemma 9.3. Let $\mathscr{A}=\cup\left\{A_{\mu}: \mu \in \mathscr{C}\right\}$ be a locally finite system of $F_{\sigma}$-sets in a space $X$. Then the set $A=\cup\left\{A_{\mu}: \mu \in \mathscr{C}\right\}$ is also a $F_{\sigma}$-set in $X$.

The lemma follows from the fact that a union of locally finite system of closed sets is also a closed set.

Proof of Theorem 9.3. We will prove this theorem by induction on $D(X)$. If $D(X)<\omega_{0}$, then $D(X)=$ Ind $X$ and our assertion is true. Let $D(X)=\beta \geqq \omega_{0}$ and the equality

$$
X=\cup\left\{A_{\delta}: \delta \leqq \alpha=J(\beta)\right\}
$$

be a $\beta$ - $D$-representation of $X$. We put

$$
Z=Y \backslash \bar{A}_{\alpha}
$$

where $\bar{A}_{\alpha}$ is a closure of $A_{\alpha}$ in $Y$. Let $U_{\delta}=X \backslash \cup\left\{A_{\delta^{\prime}}: \delta^{\prime}>\delta\right\}$ where $\delta<\alpha$. Then from condition (e) of Definition 0.3 we have

$$
X \backslash A_{\alpha}=\cup\left\{U_{\tilde{o}}: \delta<\alpha\right\}
$$

and by Lemma 8.3 in [13], Luxemburg, $U_{\delta}$ is open in $X$ and

$$
D\left(U_{\delta}\right)<\alpha=J(\beta) \leqq \beta .
$$

Let $V_{\delta}$ be an open in $Y$ set such that $V_{\delta} \subset Z$ and

$$
V_{\delta} \cap X=U_{\delta} .
$$

We put $V=\cup\left\{V_{\delta}: \delta<\alpha\right\}$ then $V \subset Z$ and $V$ is an open set in $Y$. 
Since $V$ is paracompact, we can find a closed in $V$ locally finite refinement $\mathscr{F}=\left\{F_{\mu}: \mu \in \mathscr{C}\right\}$ of the covering $\left\{V_{\dot{\delta}}: \delta<\alpha\right\}$. Consequently from (16), (17) it follows that

$$
D\left(F_{\mu} \cap X\right)<\alpha \quad(\mu \in \mathscr{C}) .
$$

By inductive assumption for every $\mu \in \mathscr{M}$ there exists a $G_{\delta}$-set $G_{\mu}$ in $F_{p}$ such that

$$
X \cap F_{\mu} \subset G_{\mu} \subset F_{r} \quad D\left(G_{\mu}\right)=D\left(F_{\mu} \cap X\right)<\alpha .
$$

Since the system $\mathscr{F}$ is locally finite in $V$, the system $\mathscr{L}=\left\{L_{\mu}=\right.$ $\left.F_{\mu} \backslash G_{\mu}: \mu \in \mathscr{C}\right\}$ is also locally finite in $V$. Moreover, since $G_{\mu}$ is a $G_{\delta}$-set in $V$ the set $L_{\mu}$ is $F_{o}$-set in $V$ and by Lemma 9.3 the set $L=$ $\cup\left\{L_{\mu}: \mu \in \mathscr{C}\right\}$ is $F_{o}$ in $V$. We put $G_{1} V \backslash L$, then clearly, $G_{1}$ is a $G_{\hat{o}}$-set in $V$ (and consequently in $Y$ ) and

$$
Z \supset G_{1} \supset X \backslash A_{\alpha} \text {. }
$$

Since $F_{\mu} \cap G_{1}=F_{\mu} \backslash L$, sets $F_{\mu} \backslash L$ are closed in $G_{1}$. Moreover, the system $\left\{F_{\mu} \backslash L, \mu \in \mathscr{C}\right\}$ is locally finite in $V$ (and consequently in $G_{1}$ ) and $F_{\nu} \backslash L \subset G_{\nu}$. Therefore

$$
D\left(F_{p} \backslash L\right) \leqq D\left(G_{\mu}\right)<\alpha .
$$

By the sum theorem for locally finite union of closed sets (see [3], Henderson) we get

$$
D\left(G_{1}\right)=D\left(\cup\left\{F_{\mu} \backslash L: \mu \in \mathscr{C}\right\}\right) \leqq \sup \left\{D\left(F_{\mu} \backslash L\right): \mu \in \mathscr{C}\right\} \leqq \alpha=J(\beta) .
$$

Let us consider the set $A_{\alpha} \subset Y \backslash Z \subset Y \backslash G_{1}$. Since Ind $A_{\alpha} \leqq K(\beta)<\omega_{0}$ (see condition (d) of Definition 0.3 ) there exists a $G_{\delta}$-set $G_{2}$ in $Y$ such that

$$
A_{\alpha} \subset G_{2}, \quad D\left(A_{\alpha}\right)=\text { Ind } A_{\alpha}=D\left(G_{2}\right) \leqq K(\beta) .
$$

We put $G_{3}=G_{2} \cap(Y \backslash Z)$, then clearly $G_{3}$ is closed in $G=G_{3} \cup G_{1}$ and $G_{3}$ is a $G_{\delta}$-set in $Y$, because $Y \backslash Z$ is a closed set. Moreover, we have obviously that

$$
X \subset G, \quad D\left(G_{3}\right) \leqq D\left(G_{2}\right) \leqq K(\beta)
$$

and $G$ is a $G_{\delta}$-set in $Y$ as a union of $G_{\tilde{\delta}}$-sets $G_{1}$ and $G_{3}$. Since $G_{3}$ is closed in $G$, the set $G_{1}=G \backslash G_{3}$ is open in $G$ and consequently (see [3]) from (18), (19) it follows that:

$$
D(G) \leqq D\left(G_{1}\right)+D\left(G_{3}\right) \leqq J(\beta)+K(\beta)=\beta=D(X) .
$$

The inequality $D(X) \leqq D(G)$ follows from the inclusion $X \subset G$.

10. On the necessary and sufficient conditions of the existence 
of transfinite dimensions. As it was mentioned in the introduction, dimensions ind $X$ and Ind $X$ do not exist for every space $X$. In this section we shall consider that $D$-dimension of a space $X$ exists if $D(X)$ is an ordinal number. The following theorem gives a criterion of the existence of transfinite dimensions in terms of compactifications.

THEOREM 10.1. Let $X$ be a separable space, then

(a) Ind $X$ exists $\Leftrightarrow$ there is a countable dimensional ${ }^{17}$ compactification $c X \supset X$ such that $P(c X)=P(X)$.

(b) ind $X$ exist $\Leftrightarrow$ there is a countable dimensional compactification $c X \supset X$.

(c) $D(X)$ exists $\Leftrightarrow$ there is a weakly countable dimensional compactification $c X \supset X$.

To prove this we need the following assertions:

(1) If Ind $X$ exists then $X$ is countable dimensional, see [21], Smirnov.

(2) If $X$ is a countable dimensional compactum then Ind $X$ exists, see [22], Smirnov.

(3) If $X$ is a complete countable dimensional space then ind $X$ exists, see [5], Hurewicz and Wallman.

(4) If ind $X$ exists, $X$ is a separable space, then $X$ is countable dimensional, see [5], Hurewicz and Wallman.

(5) If $X$ is a complete separable space, then $D(X)$ exists $\Leftrightarrow$ is a weakly countable dimensional ${ }^{18}$, see [4], Henderson.

Proof of Theorem 10.1. (a) Let Ind $X$ exist, then the existence of such a compactification $c X$ follows from Corollary 1.1 and (1). If there exists a countable dimensional compactification $c X \supset X$ then by virtue of (2) Ind $X$ exists and our assertion follows from Lemma 8.2.

(b) Let ind $X$ exist, then by Corollary 9.2 there exists a separable absolute $G_{\delta}$-set $p X \supset X$ such that ind $p X=$ ind $X$. Since $p X$ is an absolute $G_{j}$-set there exists a compactification $c X \supset p X$ such that the set $c X \backslash p X$ is countable dimensional see [9], Lelek. Since ind $p X$ exists, by virtue of (4), the space $p X$ is countable dimensional, consequently the compactum $c X$ is also countable dimensional. If a space $X$ has a countable dimensional compactification $c X \supset X$, then by virtue of (3) ind $c X$ exists. Consequently, ind $X$ also exists (see (1), introduction).

Property (c) follows from (5), Theorem 8.1 and (1) introduction.

${ }^{17}$ A space $X$ is countable dimensional if $X$ is a union of countable number of zero-dimensional sets.

18 It is easy to prove this theorem also for nonseparable spaces. 
Corollary 10.1. For any separable space $X$ with dimension ind $X$ there exists a compactification $c X$ such that the dimension ind $c X$ also exists. This corollary follows from Theorem 10.1(b) and (3).

Theorem 7.1 shows that we can not require that the equality ind $c X=$ ind $X$ holds for some compactification $c X \supset X$. However I think that the technique of the proof of Theorem 1.1 will permit proving the following.

Conjecture. If $X$ is a separable space and ind $X=\alpha+p$, where $\alpha=J(\alpha+p), p=K(\alpha+p)=0,1,2, \cdots$, then there exists a compactification $c X \supset X$ such that ind $c X \leqq \alpha+2 p+1$.

We also note that using Theorems 9.1 and 9.3 we can easily obtain criteria of the existence of dimensions Ind $X$ and $D(X)$ in terms of completions for nonseparable spaces.

\section{REFERENCES}

1. H. G. Bothe, Eine Einbettung $m$-dimensionaler Mengen in einen $(m+l)$-dimensionalen absoluten Retrakt, Fund. Math., 52 (1963), 209-224.

2. S. Hanai, and K. Morita, Closed mappings and metric spaces, Proc. Japan Acad., 32 (1956), 10-14.

3. D. W. Henderson, D-dimension, I. A new transfinite dimension, Pacific J. Math., 26 (1968), 91-107.

4. - D-dimension, II. Separable spaces and compactifications, Pacific J. Math., 26 (1968), 109-113.

5. W. Hurewicz and H. Wallman, Dimension Theory, Princeton Math., Ser. vol. 4, Princeton Univ. Press, Princeton, N. J., 1941.

6. M. Katetov, On the dimension of non-separable spaces $I$, (Russian), Czechoslovak. Mat. J., 2 (77) (1952), 333-368 (1953). (Russian, English summary).

7. I. M. Kozlovsky, Two theorems on metric spaces, (Russian), Dokl. Akad. Nauk SSSR., 204 (1972), 784-787 = Soviet Math. Dokl., 13 (1972), 743-747.

8. K. Kuratowski, Topology, Vol. I. New edition. Academic Press, New York-London, Panstwowe Wydawnictwo Naukowe, Warsaw, 1966, xx +560 .

9. A. Lelek, On the dimensionality of remainders in compactifications, (Russian), Dokl. Acad. Nauk SSSR., 160 (1965), 534-537.

10. B. T. Levsenko, Spaces of transfinite dimension, (Russian), Mat. Sb. (N. S.), 67 (109) (1965), 255-266.

11. L. A. Luxemburg, On transfinite D-dimension, Theses of the 6th All Union Topolo. gical Conference, Tbilisi, 1972 (Russian).

12. - On compact spaces with noncoinciding transfinite dimensions, Dokl. Akad. Nauk SSSR., TOM 212 (1973) No. 6 (Russian) = Soviet Math. Dokl., 4 (1973) No. 5 , 1593-1597.

13. - On compact metric spaces with noncoinciding transfinite dimensions, Pacific J. Math., 93 (1981), 339-386.

14. - On transfinite inductive dimensions, Dokl. Akad. Nauk SSSR., 209 (1973)

No.6, 295-298 (Russian) = Soviet Math. Dokl., 14 (1973), 388-393.

15. J. I. Nagata, Modern Dimension Theory, Bibliotheca Mathematica, vol. VI. Interscience Publishers, [John Wiley and Sons, Inc.] New York, 1965. 
16. B. A. Pasynkov, Partial topological products, (Russian), Trudy Moskov. Mat. Obsc., 13 (1965), 136-245.

17. B. A. Pasynkov, The dimensionality of normal spaces, (Russian), Dokl. Akad. Nauk SSSR., 201 (1971), 1049-1052 = Soviet Math. Dokl., 12 (1971), 1784-1787.

18. P. Roy, Failure of equivalence of dimension concepts for metric spaces, Bull. Amer. Math. Soc., 68 (1962), 609-613.

19. E. G. Sklyarenko, Dimensionality properties of infinite dimensional spaces, (Russian), Izv. Akad. Nauk SSSR. Ser. Mat., 23 (1959), 1203-1206. Amer. Math. Transl., (2) 21 (1962), 25-50.

20. Ju. M. Smirnov, Universal spaces for certain classes of infinite-dimensional spaces, (Russian), Izv. Akad. Nauk SSSR. Ser. Mat., 23 (1959), 185-196; English transl., Amer. Math. Soc. Transl., (2) 21 (1962), 21-33.

21. Yu. M. Smirnov, Some remarks on transfinite dimension, (Russian), Dokl. Akad. Nauk SSSR., 141 (1961), 814-817.

22. - On transfinite dimension, Matem. Sbornik., 58 No. 4 (1962), 415-422 (Russian).

23. A. Zarelua, The Equality of dimensions and bicompact extensions, (Russian), Dokl. Akad. Nauk SSSR., 144 (1962), 713-716.

Received March 5, 1979 and in revised form February 12, 1981.

UnIVERSity OF KentuCKY

Lexington, KY 40506 


\section{PACIFIC JOURNAL OF MATHEMATICS}

\section{EDITORS}

DONALD BABBITT (Managing Editor)

University of California

Los Angeles, CA 90024

Hugo Rossi

University of Utah

Salt Lake City, UT 84112

C. C. MOore and Arthur Agus

University of California

Berkeley, CA 94720
J. DugundJI

Department of Mathematics

University of Southern California

Los Angeles, CA 90007

R. FinN and J. MILGRAM

Stanford University

Stanford, CA 94305

\section{ASSOCIATE EDITORS}
R. ARENS
E. F, BECKENBACH
B. H. NeumanN
F. WOLF
K. YoSHIDA

\section{SUPPORTING INSTITUTIONS}

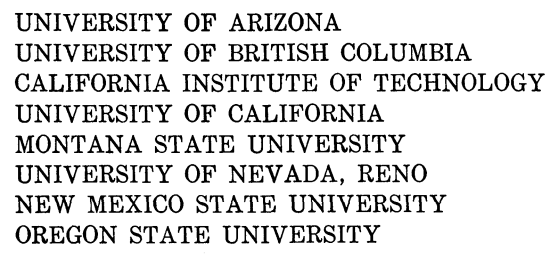

UNIVERSITY OF ARIZONA

UNIVERSITY OF BRITISH COLUMBIA

CALIFORNIA INSTITUTE OF TECHNOLOGY

UNIVERSITY OF CALIFORNIA

MONTANA STATE UNIVERSITY

UNIVERSITY OF NEVADA, RENO

NEW MEXICO STATE UNIVERSITY

OREGON STATE UNIVERSITY

\author{
UNIVERSITY OF OREGON \\ UNIVERSITY OF SOUTHERN CALIFORNIA \\ STANFORD UNIVERSITY \\ UNIVERSITY OF AAWAII \\ UNIVERSITY OF TOKYO \\ UNIVERSITY OF UTAH \\ WASHINGTON STATE UNIVERSITY \\ UNIVERSITY OF WASHINGTON
}

The Supporting Institutions listed above contribute to the cost of publication of this Journal, but they are not owners or publishers and have no responsibility for its content or policies,

Mathematical parers intended for publication in the Pacific Journal of Mathematics should be in typed form or offset-reproduced, (not dittoed), double spaced with large margins. Please do not use built up fractions in the text of the manuscript. However, you may use them in the displayed equations. Underline Greek letters in red, German in green, and script in blue. The first paragraph or two must be capable of being used separately as a synopsis of the entire paper. Please propose a heading for the odd unmbered pages of less than 35 characters. Manuscripts, in triplicate, may be sent to any one of the editors. Please classify according to the scheme of Math. Reviews, Index to Vol. 39. Supply name and address of author to whom proofs should be sent. All other communications should be addressed to the managing editor, or Elaine Barth, University of California, Los Angeles, California, 90024.

50 reprints to each author are provided free for each article, only if page charges have been substantially paid. Additional copies may be obtained at cost in multiples of 50 .

The Pacific Journal of Mathematics is issued monthly as of January 1966, Regular subscription rate: $\$ 114.00$ a year $(6$ Vol., 12 issues). Special rate: $\$ 57.00$ a year to individual members of supporting institution.

Subscriptions, orders for numbers issued in the last three calendar years, and changes of address shoud be sent to Pacific Journal of Mathematics, P.O. Box 969, Carmel Valley, CA 93924, U.S.A. Old back numbers obtainable from Kraus Periodicals Co., Route 100, Millwood, NY 10546.

PUBLISHED BY PACIFIC JOURNAL OF MATHEMATICS, A NON-PROFIT CORPORATION

Printed at Kokusai Bunken Insatsusha (International Academic Printing Co., Ltd.). 8-8, 3-chome, Takadanobaba, Shinjuku-ku, Tokyo 160, Japan.

Copyright (C) 1982 by Pacific Journal of Mathematics Manufactured and first issued in Japan 


\section{Pacific Journal of Mathematics}

\section{Vol. 101, No. 2 December, 1982}

Jean Bourgain, A Hausdorff-Young inequality for $B$-convex Banach

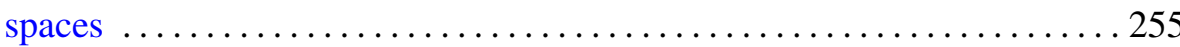

J. L. Brenner and Lorraine L. Foster, Exponential Diophantine

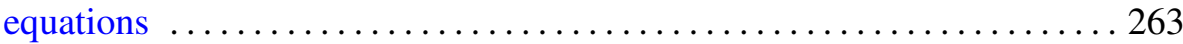

Henry H. Glover and William Duncan Homer, II, Fixed points on flag

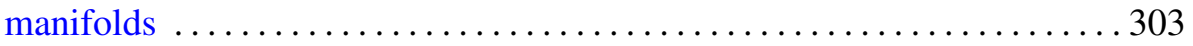

Lothar Hahn, A note on stochastic methods in connection with approximation theorems for positive linear operators $\ldots \ldots \ldots \ldots \ldots . \ldots 307$

James P. Henderson, Approximating cellular maps between low-dimensional polyhedra

V. K. Jain, Certain transformations of basic hypergeometric series and their

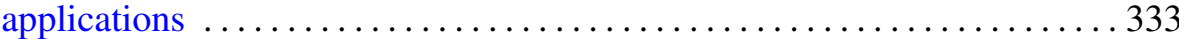

Charles David Keys, On the decomposition of reducible principal series

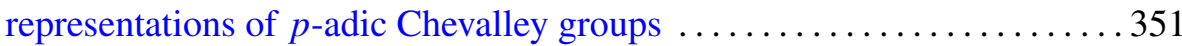

M. S. Klamkin and A. Meir, Ptolemy's inequality, chordal metric,

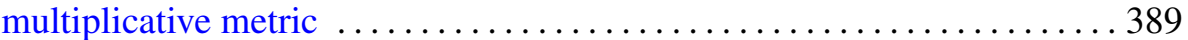

Robert F. Lax, Independence of normal Weierstrass points under

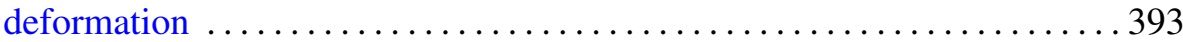

Leonid A. Luxemburg, On compactifications of metric spaces with

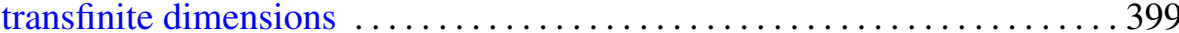

Carlton James Maxson, Martin Ross Pettet and Kirby C. Smith, On semisimple rings that are centralizer near-rings .....

Teodor C. Przymusiński, Extending functions from products with a metric factor and absolutes

Giorgio Talenti, A note on the Gauss curvature of harmonic and minimal surfaces

D. M. Terlinden, A spectral containment theorem analogous to the semigroup theory result $e^{t \sigma(A)} \subseteq \sigma\left(e^{t A}\right)$ 